University of Rhode Island

DigitalCommons@URI

Civil \& Environmental Engineering Faculty

Publications

Civil \& Environmental Engineering

$1-28-2020$

\title{
Cooling rate effects on the structure of 45S5 bioglass: Insights from experiments and simulations
}

Pratik Bhaskar

Rajesh Kumar

Yashasvi Maurya

R. Ravinder

Amarnath R. Allu

See next page for additional authors

Follow this and additional works at: https://digitalcommons.uri.edu/cve_facpubs

The University of Rhode Island Faculty have made this article openly available.

Please let us know how Open Access to this research benefits you.

This is a pre-publication author manuscript of the final, published article.

Terms of Use

This article is made available under the terms and conditions applicable towards Open Access Policy Articles, as set forth in our Terms of Use.

\section{Citation/Publisher Attribution}

Bhaskar, P., Kumar, R., Maurya, Y., et al. (2020). Cooling rate effects on the structure of 45S5 bioglass:

Insights from experiments and simulations. Journal of Non-crystalline Solids, 534, 119952.

https://doi.org/10.1016/j.jnoncrysol.2020.119952

This Article is brought to you for free and open access by the Civil \& Environmental Engineering at DigitalCommons@URI. It has been accepted for inclusion in Civil \& Environmental Engineering Faculty Publications by an authorized administrator of DigitalCommons@URI. For more information, please contact digitalcommonsgroup@uri.edu. 


\section{Authors}

Pratik Bhaskar, Rajesh Kumar, Yashasvi Maurya, R. Ravinder, Amarnath R. Allu, Sumanta Das, Nitya Nand Gosvami, Randall E. Youngman, Mikkel S. Bodker, Nerea Mascaraque, Morten M. Smedskjaer, Mathieu Bauchy, and N.M. Anoop Krishnan 


\title{
Cooling Rate Effects on the Structure of 45S5 Bioglass: Insights from Experiments and Simulations
}

Pratik Bhaskar ${ }^{1, \#}$, Yashasvi Maurya ${ }^{1, \#}$, Rajesh Kumar ${ }^{1}$, R. Ravinder ${ }^{1}$, Amarnath R. Allu ${ }^{2}$, Sumanta Das ${ }^{3}$, Nitya Nand Gosvami $^{4}$, Randall E. Youngman ${ }^{5}$, Mikkel S. Bødker ${ }^{6}$, Nerea Mascaraque ${ }^{6}$, Morten M. Smedskjaer ${ }^{6}$, Mathieu Bauchy ${ }^{7, *}$, N. M. Anoop Krishnan ${ }^{1,4, *}$

\footnotetext{
${ }^{1}$ Department of Civil Engineering, Indian Institute of Technology Delhi, Hauz Khas, New Delhi 110016, India

${ }^{2}$ Glass Division, CSIR-Central Glass and Ceramic Research Institute, 700032, Kolkata, India

${ }^{3}$ Department of Civil and Environmental Engineering, University of Rhode Island, Kingston, RI, USA

${ }^{4}$ Department of Materials Science and Engineering, Indian Institute of Technology Delhi, Hauz Khas, New Delhi 110016, India

${ }^{5}$ Science and Technology Division, Corning Incorporated, Corning, NY 14831, USA

${ }^{6}$ Department of Chemistry and Bioscience, Aalborg University, 9220 Aalborg, Denmark

${ }^{7}$ Physics of AmoRphous and Inorganic Solids Laboratory (PARISlab), Department of Civil and Environmental Engineering, University of California, Los Angeles, CA 90095, USA
}

*Corresponding author: M. Bauchy (bauchy@ucla.edu), N. M. A. Krishnan (krishnan@iitd.ac.in)

"Both the authors contributed equally.

\begin{abstract}
Due to its ability to bond with living tissues upon dissolution, 45S5 bioglass and related compositions materials are extensively used for the replacement, regeneration, and repair of hard tissues in the human body. However, the details of its atomic structure remain debated. This is partially due to the non-equilibrium nature of glasses, as their non-crystalline structure is highly dependent on their thermal history, namely, the cooling rate used during quenching. Herein, combining molecular dynamics (MD) simulations with cooling rates ranging over several orders of magnitude and experimental studies using nuclear magnetic resonance (NMR), we investigate the structure of the nominal 4555 bioglass composition. These results suggest that the MD simulation results when extrapolated to experimental cooling rates can provide a reasonable to estimate of the structure of 45S5 bioglass. Finally, based on these results, we suggest the propensity of phosphate group to form isolated orthophosphate species. Overall, these results reconcile the simulation and experimental results on the structure of 45S5 bioglass, and particularly on the speciation of phosphate group, which may be key in controlling the bioactivity of $45 \mathrm{~S} 5$ bioglass.
\end{abstract}

\section{Introduction}

Since its introduction by Hench, $45 \mathrm{~S} 5$ bioglass remains one of the most used bioactive glass compositions [1]. 45S5 bioglass, henceforth referred to as bioglass, is constituted by the oxides of calcium, sodium, phosphorus, and silicon and has the capacity to form strong interfacial bonds with hard tissues in vivo through dissolution of ions when they are hydrated by body fluids [1]. Bioactive glass compositions have been widely used for a variety of applications ranging from scaffolds for tissue engineering,[2] bone grafts, [3] periodontal applications,[4,5] and even as a coating for bioinert implants.[6,7] Despite the wide usage of bioactive glasses, some open questions regarding the atomic-level structure and other structural features of bioglass remain, which largely arise from some inconsistencies between previously reported simulation and experiment results.[8-29] This is exemplified by the fact that in bioglass, while 
experimental studies suggest that phosphate species exist majorly as isolated tetrahedra,[8,9,26-30] atomistic simulations suggest the existence of phosphate tetrahedra with higher degrees of crosslinking[9-12,15,19,20]. A detailed understanding of the structure of bioglass is essential to tailor the dissolution processes governing bioactivity.[26,27,31]

Bioglass samples, like other glasses, are out-of-equilibrium systems formed by the rapid quenching of an isochemical equilibrium liquid.[32,33] Accordingly, the structure and properties of bioglass strongly depend on the thermal and pressure history they have experienced during melt-quenching or subsequent processing. The structure of bioglasses is typically characterized using techniques such as infrared spectroscopy, Raman spectroscopy, magic angle spinning nuclear magnetic resonance (MASNMR) spectroscopy,[26-28] or using computational techniques such as molecular dynamics (MD) or density function theory (DFT) simulations. $[8,9,19]$ MD simulations are widely used as they provide some insights into the atomic details that are otherwise invisible to conventional experiments. In particular, classical MD simulations are usually preferred over DFT simulations due to the latter's limitation to small sizes (typically $<500$ atoms) and timescales (typically $<10$ picosecond). Although classical MD simulations are also restricted to short timescales (typically a few nanoseconds), this timescale can be four to five orders of magnitude higher than that of DFT simulations. In turn, these short timescales (both for MD and DFT) lead to unrealistically high cooling rates in simulations, such as $10^{12} \mathrm{~K} / \mathrm{s}$, which is almost 10 orders of magnitude higher than typical experimental cooling rates (1-to- $100 \mathrm{k} / \mathrm{s}$ ). Therefore, understanding the effect of the cooling rate on the structure of bioglass provides the key for establishing a realistic, unified description of the atomic structure of bioglass.

Several earlier studies have been conducted to study the effect of cooling rates on model glasses, [3436 ] vitreous silica,[37-39] sodium silicate glasses[40,41], and $45 S 5$ bioglass[10,15] structures. The high cooling rate used in simulations typically results in more structural defects.[40,42,43] Further, the cooling rate is found to have a notable impact on some of the macroscopic properties such as density, thermal expansion, and elastic modulus[40,44]-wherein the extent of the dependence on the cooling rate depends on the glass composition. Moreover, earlier studies based on MD simulations have suggested that, although the short range order is usually weakly affected, the medium range order can present notable changes with varying cooling rates.[40] This is important as, in the case of bioglass, the medium range order is suggested to play a major role in controlling the bioactivity.[27] This raises some concerns on the validity of the structure-property relations generated by MD simulations.

Extensive studies has have been conducted in the past to analyze the structure of 45S5 bioglass [8-28]. In particular, the phosphorus speciation has been debated when reconciling experimental and simulation studies. Experimental studies suggest that the majority of the phosphate species present in the bioglass exist as isolated orthophosphate units, although non-negligible amount of $Q_{p}{ }^{1}$ species have been observed [16,20,23-26]. In some studies, ${ }^{31} \mathrm{P}$ MAS-NMR spectroscopy revealed a broad resonance at around 9 ppm corresponding to isolated orthophosphate anions $\left(\mathrm{PO}_{4}{ }^{3-}\right)[26,28,30]$. These isolated orthophosphate anions associate with metal cations, thereby reducing the network-modifying role of $\mathrm{Ca} / \mathrm{Na}$ cations in the silicate network.[28] However, multiple molecular dynamics (MD) $[9-11,13,15]$ and ab-initio simulation $[12,20,22]$ studies have confirmed the existence of non-orthophosphate groups. The MD studies have been conducted using rigid-ion $[15,45]$ and core-shell potentials $[10,11]$, with the core- 
shell potential leading to lower non- $Q_{p}^{0}$ species in comparison to rigid-ion one. While there has been some cooling rate studies using MD simulations on the $Q^{n}$ speciation using both rigid-ion $[15,45]$ and core-shell models [10], no clear picture of the nature of $P$ speciation has emerged $[10,11]$. To reconcile the experimental and simulation results, a later study suggested that, although it is possible that $P$ may exist as $Q^{1}$ or $Q^{2}$ species, the levels of these species must be significantly lower than those suggested by MD simulations [26].

Herein, using MD simulations, we investigate the structure of bioglass samples prepared using cooling rates ranging over five orders of magnitudes. In line with previous results, $[10,40]$ we find that the cooling rate strongly affects the medium-range order structure, whereas the short-range order remains fairly unaffected. Interestingly, we observe of an increasing propensity phosphate group to exist as isolated orthophosphate ions upon decreasing cooling rate. Finally, by extrapolating the structural features obtained from MD simulations such as $Q^{n}$ distribution to experimental cooling rates, we demonstrate that MD simulations can be reconciled with our NMR measurements of $Q^{n}$ speciation to provide a consistent structure for bioactive glasses.

\section{Methodology \\ Simulation details}

\section{(i) Glass simulation}

$45 \mathrm{~S} 5$ bioglass samples, with the nominal molar composition $(\mathrm{CaO})_{26.9}$. $\left(\mathrm{Na}_{2} \mathrm{O}\right)_{24.4 .}\left(\mathrm{SiO}_{2}\right)_{46.1}$. $\left(\mathrm{P}_{2} \mathrm{O}_{5}\right)_{2.6}$, were prepared using classical MD simulations by applying the traditional "melt-quench" method.[40,46,47] All simulations were performed using the open-source LAMMPS (Large-scale Atomic/Molecular Massively Parallel Simulator) package.[48] In order to study the effect of the cooling rate, the glass samples were prepared with cooling rates varying over five orders of magnitudes, from $100 \mathrm{~K} / \mathrm{ps}$ to 0.01 $\mathrm{K} / \mathrm{ps}$. The interactions between the atoms were simulated using the well-established Teter potential, $[8,40]$ which has a Buckingham-like form. This potential has been extensively validated against experimental structure data for a wide-range of silicate and phosphate minerals and glasses, [40,44,4951] including $45 S 5$ bioglass (see Supplementary Information and Ref.[8]). A repulsive term, $V(r)=B / r^{n}+$ $D r$, was adopted for $r$ smaller than $r_{0}$ (variable for each of the atom pairs), to avoid the "Buckingham catastrophe" at high temperature. Here, $r_{0}$ is defined as the value of $r$ when the third derivative of potential energy approaches zero. At $r_{0}, B$ and $n$ were chosen to make potential energy and its derivatives continuous. The long range Coulombic interactions were calculated with the particle-particle particlemesh (PPPM) solver algorithm with an accuracy of $10^{-5}$.

Initial configurations were built by randomly placing 5673 atoms in a cubical box such that there is no unrealistic overlap. The system was then equilibrated for $1 \mathrm{~ns}$ at $3000 \mathrm{~K}$ and zero pressure in the isothermal isobaric (NPT) ensemble. This was to ensure that the system reaches an equilibrium state, without any memory of the initial structure obtained by the random placement of atoms. After equilibration, glasses were formed by gradually cooling the systems from 3000 to $300 \mathrm{~K}$ linearly at five different cooling rates, namely, $100,10,1,0.1$, and $0.01 \mathrm{~K} / \mathrm{ps}$ under zero pressure in the NPT ensemble. Note that continuous cooling, with no intermittent equilibration, was carried out from 3000 to $300 \mathrm{~K}$ using the Nose-Hoover thermostat [52]. Finally, all the glass structures were relaxed for $100 \mathrm{ps}$ at $300 \mathrm{~K}$ and zero pressure in the NPT ensemble. For statistical averaging, the final glass structure obtained was equilibrated in an NVT ensemble for $100 \mathrm{ps}$. All the structural and thermodynamic data of the final glass 
structure were collected during this run. All the properties referring to the "glassy state" are obtained by averaging over 100 frames extracted from this run.

\section{(ii) Pair distribution function}

The pair distribution function (PDF) represents the ratio of the local density of atoms with respect to the global density of the atoms in terms of distance from an atom. In particular, the neutron pair distribution function is given by:

$$
g_{N}(r)=\frac{1}{\sum c_{i} c_{j} b_{i} b_{j}} \sum c_{i} c_{j} b_{i} b_{j} g_{i j}(r),
$$

where $c_{i}$ is the fraction of $i$ atoms $(i=\mathrm{Na}, \mathrm{Ca}, \mathrm{Si}, \mathrm{P}$, or $\mathrm{O}), b_{i}$ is the neutron scattering length of the species, and $g_{i j}$ are the partial PDFs. Using the partial PDF, the coordination number of each of the species can be obtained by computing the number of neighbors within the first coordination shell of the respective atoms. The cutoff distance was obtained from the first minimum of the respective partial PDFs.

\section{(iii) Structure factor}

The partial structure factors were calculated from the Fourier transformation of the partial pair distribution functions $g_{i j}(r)$ as:

$$
S_{i j}(Q)=1+\rho_{0} \int 4 \pi r^{2}\left[g_{i j}(r)-1\right]\left(\sin \left(Q_{r}\right) / Q_{r}\right)(\sin (\pi r / R) /(\pi r / R)) d r, \quad \text { Equation (2) }
$$

where $Q$ is the scattering vector, $\rho_{0}$ is the average atom number density, and $R$ is the maximum value of the integration in real space, which is set to half of the size of one side of the simulation cell. The total structure factor was calculated as:

$$
S_{N}(Q)=\frac{1}{\left(\sum c_{j} c_{i} b_{j} b_{i}\right)^{2}} \sum c_{i} c_{j} b_{i} b_{j} S_{i j}(Q),
$$

where $c_{i}$ and $c_{j}$ are the fractions of atoms and $b_{i}$ and $b_{j}$ are neutron scattering lengths, for elements $i$ and $j$, respectively. The partial pair distribution functions and structure factors were plotted by taking statistical averages over 100 frames at $300 \mathrm{~K}$. The position of the first sharp diffraction peak (FSDP) and the full width at half maxima (FWHM) of the structure factors were calculated by fitting the FSDP with a Lorentzian function for all the cooling rates.

\section{(iv) $Q^{\text {n }}$ distribution}

The $Q^{n}$ notation quantifies the degree of connectivity of the tetrahedra present in oxide glass networks. In this $\mathrm{Q}^{n}$ notation, $n$ represents the number of oxygens that form a bridge between two tetrahedranamely, bridging oxygen (BO)-around a network-forming cation. Thus, $\mathrm{Q}^{4}$ represents a tetrahedron connected to four other tetrahedra through BO atoms. For $45 \mathrm{~S} 5$ bioglass, the tetrahedra can be formed by $\mathrm{SiO}_{4}$ and $\mathrm{PO}_{4}$ species. Here, the $\mathrm{Q}^{\mathrm{n}}$ distribution was computed through an in-house code, which counts the number of $\mathrm{BO}$ associated with each Si/P atoms.

\section{(v) Ring distribution}

In glassy networks, a ring represents a closed path made of interatomic bonds. In particular, we focused on hetero-polar primitive rings that represent the shortest path made of $\mathrm{Si}-\mathrm{O}$ and $\mathrm{P}-\mathrm{O}$ bonds to return to the starting atom without retracing any route. The open-source RINGS package was used to compute the ring size distribution.[53] Note that in oxide glasses, the ring size is represented by the number of 
network formers in the ring, in this case, Si or P. Thus, a ring size of $m$ contains $2 m$ atoms, namely, with $m \mathrm{O}$ atoms and $m$ Si or $\mathrm{P}$ atoms.

\section{Experimental details}

\section{(i) Glass preparation}

The 45S5 glass was prepared using the melt-quenching method by mixing a batch of the following reagent grade materials: $\mathrm{Na}_{2} \mathrm{CO}_{3}$ (Ph Eur. $\geqslant 99.5 \%$, Sigma-Aldrich), $\mathrm{CaCO}_{3}$ (Reag. Ph Eur, Merck), $\mathrm{Ca}\left(\mathrm{H}_{2} \mathrm{PO}_{4}\right)_{2} . \mathrm{H}_{2} \mathrm{O}$ (Budenheim Ibérica S.L.U., $\geqslant 95 \%$ ), and $\mathrm{SiO}_{2}$ (Sigma-Aldrich, purum p.a.). This batch, with its stoichiometric amounts, was melted in a Pt-Rh crucible at $1350^{\circ} \mathrm{C}$ for $1 \mathrm{~h}$ in an electrically heated furnace. The melt was cast onto brass plates and the transparent glass was annealed for 30 minutes at its glass transition temperature $\left(T_{\mathrm{g}}\right)$ of $523^{\circ} \mathrm{C}$. X-ray diffraction analysis shows no signs of crystallization.

\section{(ii) NMR experiments}

${ }^{29} \mathrm{Si}$ MAS NMR was conducted at $11.7 \mathrm{~T}$ (99.27 MHz resonance frequency) using an Agilent DD2 spectrometer and $5 \mathrm{~mm}$ MAS NMR probe. The powdered bioglass was packed into a $5 \mathrm{~mm}$ outer diameter zirconia MAS NMR rotor, providing sample spinning of $5 \mathrm{kHz}$. ${ }^{29} \mathrm{Si} \mathrm{MAS} \mathrm{NMR} \mathrm{data} \mathrm{were} \mathrm{collected}$ using radio-frequency pulse widths of $4.8 \mu \mathrm{s}$, corresponding to a tip angle of $\pi / 2$, and with a $10 \mathrm{~s}$ recycle delay, 3000 acquisitions were collected. The ${ }^{29} \mathrm{Si} T_{1}$ value was estimated to be on the order of $1-2 \mathrm{~s}$, resulting from the incorporation of iron (as batch material impurity) in the glass sample. $A{ }^{29}$ Si MAS NMR spectrum collected with a much longer recycle delay (60s) was identical, confirming adequate relaxation time for the NMR experiments.

${ }^{31} \mathrm{P}$ MAS NMR data were collected at 16.4 T using an Agilent DD2 spectrometer, $3.2 \mathrm{~mm}$ MAS NMR probe (22 kHz sample spinning) and a resonance frequency of $283.27 \mathrm{MHz}$. 600 acquisitions were collected with a $\pi / 2$ pulse width of $3 \mu$ s and a recycle delay of $5 \mathrm{~s}$, optimized for the short ${ }^{31} \mathrm{P} T_{1}$. MAS NMR data were processed without apodization and shift referenced to tetramethylsilane and $85 \%$ phosphoric acid solution at $0.0 \mathrm{ppm}$ for ${ }^{29} \mathrm{Si}$ and ${ }^{31} \mathrm{P}$, respectively.

\section{(iii) Deconvolution of NMR results}

Spectral deconvolution was made using DMFit[54]. The phosphate site $\left(Q^{n}\right)$ concentrations were obtained from the simulations of the isotropic peaks and all associated spinning sidebands of the ${ }^{31} \mathrm{P}$ MAS spectra. The ${ }^{31} \mathrm{P}$ quantifications were used to constrain the silica sites, assuming charge neutrality in the glasses. The silica site concentrations were obtained from Gaussian simulations of the isotropic peaks and all of their associated spinning sidebands.

\section{Results and Discussion:}

\section{(i) Enthalpy and density}



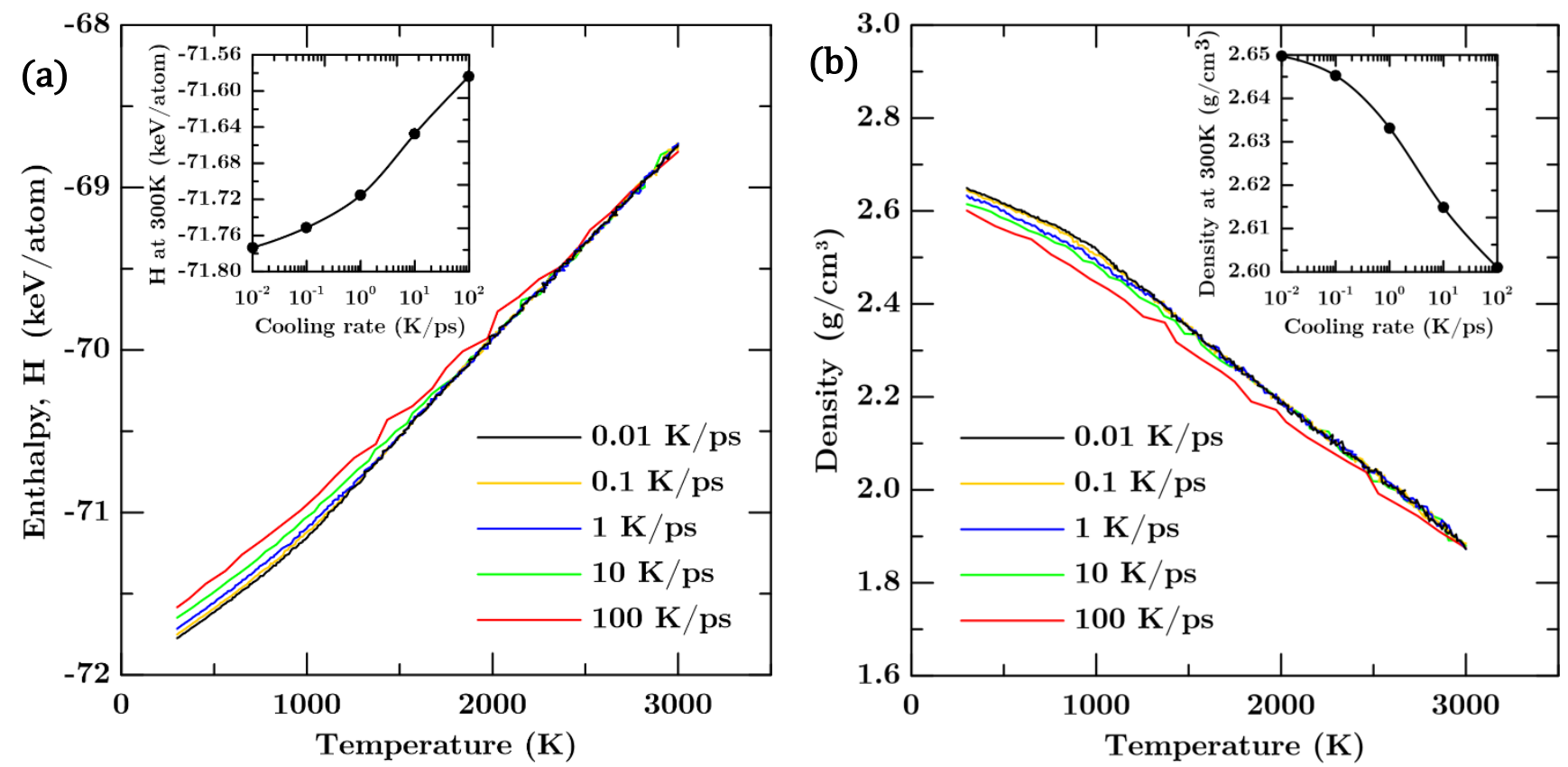

Figure 1 (a). Computed values of enthalpy $(H)$ as a function of temperature during cooling under different rates by MD simulations. The inset shows $H$ at $300 \mathrm{~K}$ as a function of the cooling rate. The line is a guide for the eye. (b) Computed values of density as a function of temperature during cooling under different rates by MD simulations. The inset shows density at $300 \mathrm{~K}$ as a function of the cooling rate. The line is a guide for the eye.

To demonstrate the effect of the cooling rate on the features of the glass transition, we are first focusing on the evolution of enthalpy $(H)$ and density of the glass upon quenching computed by MD simulations. Figure 1(a) shows the evolution of the enthalpy with respect to temperature for five different cooling rates, namely, $0.01,0.1,1,10$, and $100 \mathrm{~K} / \mathrm{ps}$. Upon quenching, we observe that the enthalpy decreases continuously and monotonically with temperature. In particular, we observe that there is no sudden change in the enthalpy of the system, confirming the absence of any first-order phase transition. Further, the enthalpy exhibits a smooth and continuous change in slope at a particular temperature, namely the "fictive temperature" of the glass[55]. Note that the fictive temperature decreases with decreasing cooling rate, in agreement with the fundamental nature of the glass transition[40,56]. The inset of Fig. 1 (a) shows the enthalpy at $300 \mathrm{~K}$ of the glasses obtained from different cooling rates. We observe that this enthalpy value decreases with decreasing cooling rate. This suggests that the glasses prepared with lower cooling rates are energetically more stable in comparison to those prepared at faster cooling rates. This can be attributed to the fact that, upon slower cooling rates, the system can sample a larger region of the energy landscape, thereby allowing it to attain lower, more stable energy states[56-59].

Figure 1(b) shows the evolution of density with respect to temperature computed by MD simulations for the five cooling rates considered. Similar to the case of the enthalpy, we observe a continuous and monotonic increase in the density of the glass with decreasing temperature-devoid of any first-order phase transition. Although a change in slope corresponding to the glass transition is also observed for the density, it does not occur at the same temperature as that of the enthalpy for each cooling rate. This is in agreement with earlier studies suggesting that the density and enthalpy relaxations in glassy 
systems at lower temperature may be decoupled[60,61]. The inset of Fig. 1(b) shows the cooling rate dependence of density of the glass at $300 \mathrm{~K}$, which increases monotonically from $2.60 \mathrm{~g} / \mathrm{cm}^{3}$ for $100 \mathrm{~K} / \mathrm{ps}$ to $2.65 \mathrm{~g} / \mathrm{cm}^{3} 0.01 \mathrm{~K} / \mathrm{ps}$, which suggest an enhanced degree of packing of the atoms in the system with slower cooling rates. Further, the simulated density of the glass tends towards the experimental value of $2.70 \mathrm{~g} / \mathrm{cm}^{3}$ with decreasing cooling rates[8]. Overall, these results confirm that our simulations offer a realistic description of the typical features of the glass transition.

(ii) Short-range order and local structure

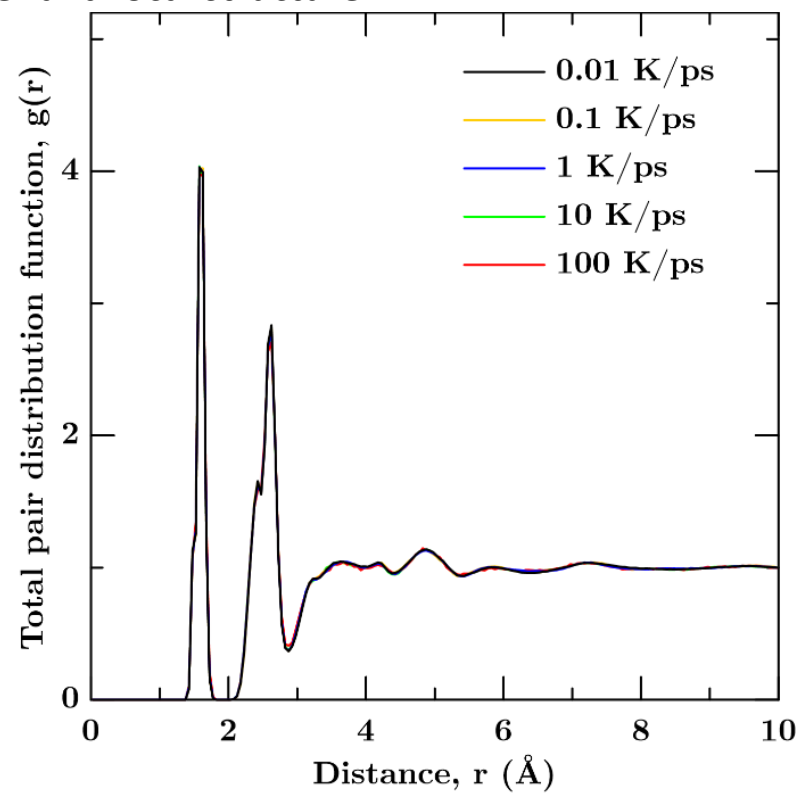

Figure 2. Total pair distribution functions in the glassy state for the studied cooling rates computed by MD simulations.
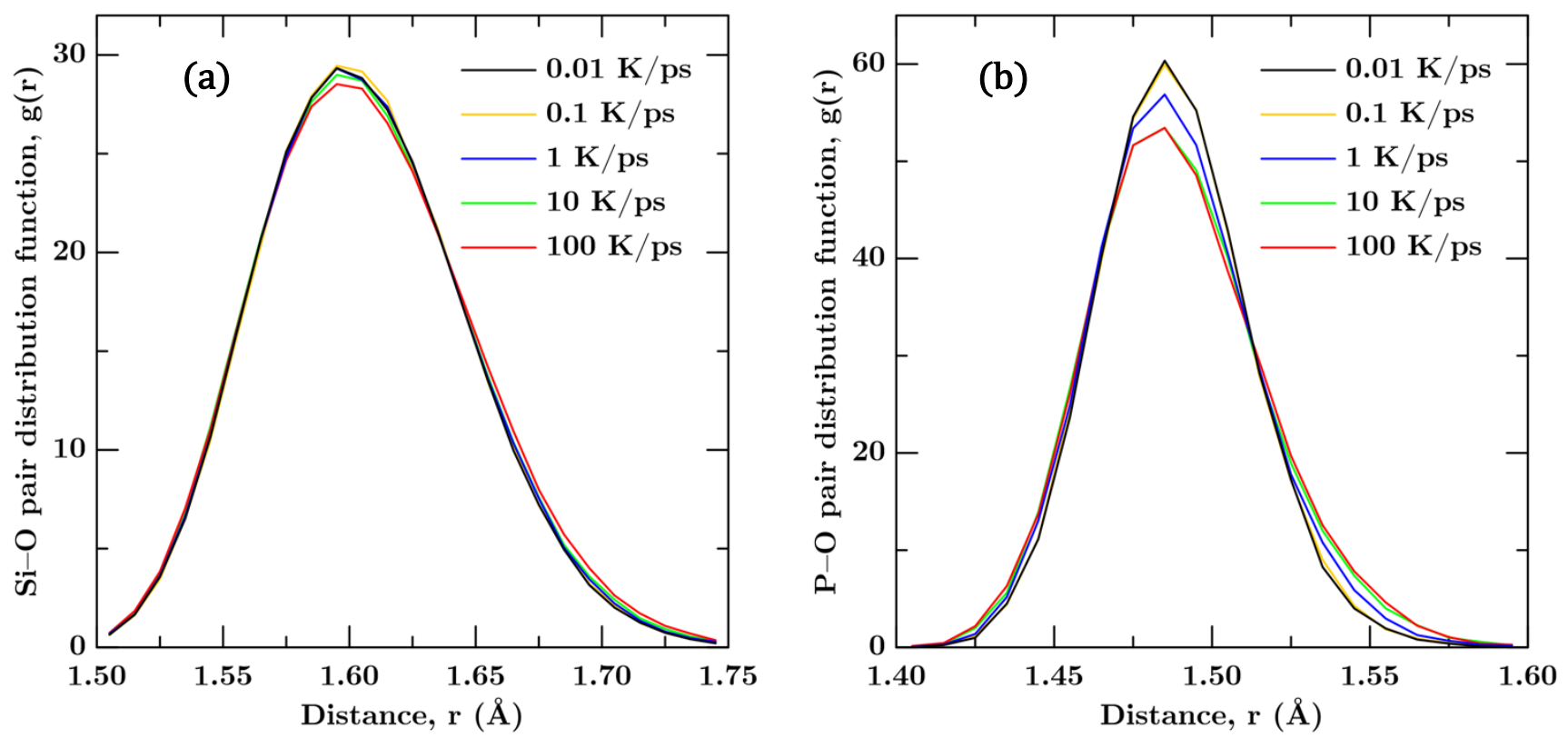

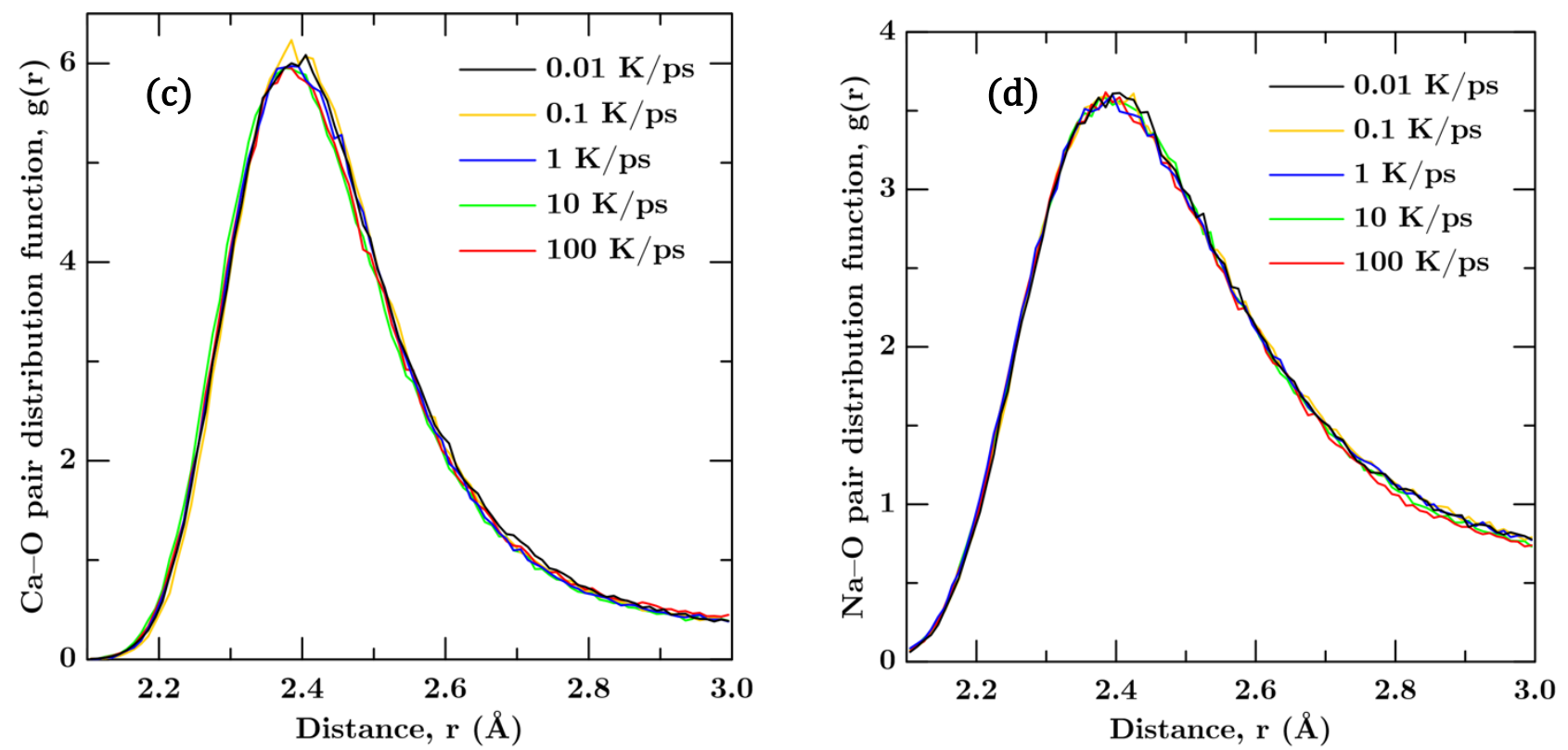

Figure 3. Hetero-nuclear partial pair distribution functions in the glassy state computed by MD simulations for the studied cooling rates of: (a) $\mathrm{Si}-\mathrm{O}$, (b) $\mathrm{P}-\mathrm{O}$, (c) $\mathrm{Ca}-\mathrm{O}$, and (d) $\mathrm{Na}-\mathrm{O}$.

Now, we analyze the short-range structure $(\leq 3 \AA)$ of the glasses obtained from MD simulations. Figure 2 shows the total pair distribution function (PDF) of the glassy structures for different cooling rates. The first peak observed around $1.5 \AA$ corresponds to the P-O interactions, which is followed by a major peak observed around $1.6 \AA$ corresponding to the $\mathrm{Si}-\mathrm{O}$ interactions. Note that the values obtained here are in agreement with previous simulations, and experiments (See Supplementary Information and Refs. $[8,9,26])$. The following peaks up to $3 \AA$ correspond to $\mathrm{Na}-\mathrm{O}, \mathrm{Ca}-\mathrm{O}$, and $\mathrm{O}-\mathrm{O}$ interactions. Beyond $3 \AA$, we observe minor peaks corresponding to second neighbor and third neighbor interactions. At larger distances, the PDF gradually converges to unity, confirming the absence of any long-range order in the system. Further, we observe that the position of the peaks of the total PDF varies only slightly, if any, with the cooling rate. This suggests that the first neighbor environments in the atomic structure are not significantly affected by the cooling rates. To further confirm this, we plot the $\mathrm{X}-\mathrm{O}$ partial PDFs (with $\mathrm{X}=$ $\mathrm{Si}, \mathrm{P}, \mathrm{Na}$, and $\mathrm{Ca}$ ). In Fig. 3, we observe that for the $\mathrm{X}-\mathrm{O}$ partial PDFs, neither the position nor the width of the peaks, are affected significantly by the cooling rate.

Next, we analyze the effect of cooling rate on the homo-nuclear interactions. Figure 4 shows the $X-X$ partial PDFs with $\mathrm{X}=\mathrm{Si}, \mathrm{O}, \mathrm{Na}$, and $\mathrm{Ca}$. We observe that the $\mathrm{X}-\mathrm{X}$ interactions peak generally become sharper with decreasing cooling rate. This suggests that the overall degree of order among increases with decreasing cooling rate. Although this effect is pronounced in the Si-Si partial PDF (see Fig. 4(a)), it is less pronounced in the other pairs (see Fig. 4(b)-(d)). The $\mathrm{Na}-\mathrm{Na}$ and $\mathrm{Ca}-\mathrm{Ca}$ partial PDFs exhibit broader peaks as seen in Figures 4(c) and (d), respectively. This is due to the increased mobility of these atoms, thanks to the non-directional ionic bonds they form with oxygen. Other partial PDFs also exhibit similar behavior wherein the first peak becomes increasingly sharper corresponding to a decreasing cooling rate (see Figs. S2-S5 of the Supplementary Material) suggesting an increased local order. This is in agreement with the earlier observation that the structure attains a lower energy stable state as the cooling rate decreases (see Fig. 1(a)). 

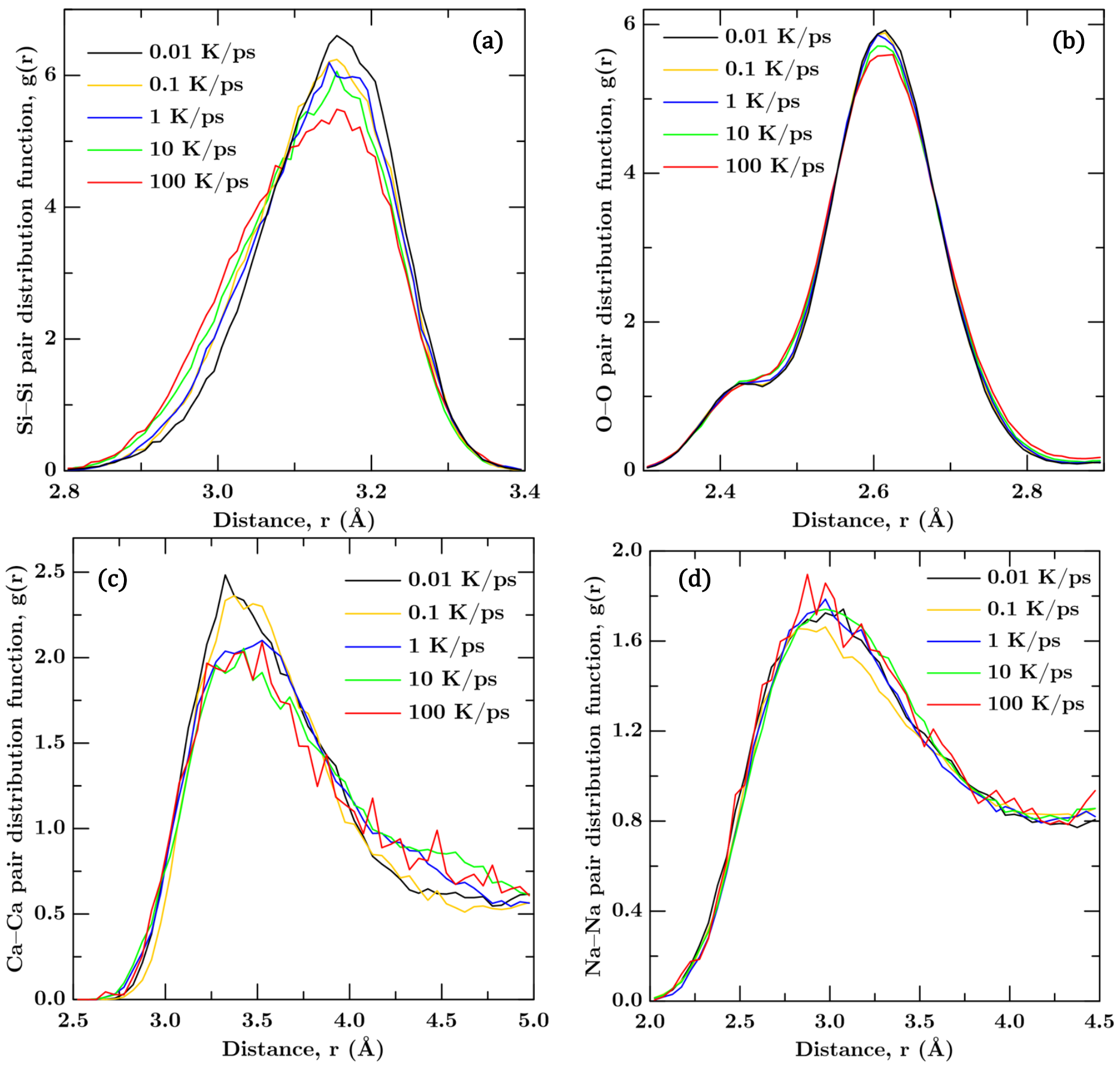

Figure 4. Homo-nuclear partial pair distribution functions in the glassy state, computed by MD simulations for the studied cooling rates, of: (a) $\mathrm{Si}-\mathrm{Si}$, (b) $\mathrm{O}-\mathrm{O}$, (c) $\mathrm{Ca}-\mathrm{Ca}$, and (d) $\mathrm{Na}-\mathrm{Na}$.

Figures 5(a) and (b) show the intra-tetrahedral O-P-O and $\mathrm{O}-\mathrm{Si}-\mathrm{O}$ bond angle distributions (BADs), respectively. We observe that the average intra-tetrahedral angles (i.e., around $109^{\circ}$ ) are not significantly affected by the cooling rate, although the peaks become slightly sharper for lower cooling rates. This suggests an increased degree of angular order upon lower cooling rate. In the case of O-P-O, 
the average bond angle decreases slightly with decreasing cooling rate up to $1 \mathrm{~K} / \mathrm{ps}$. Below $1 \mathrm{~K} / \mathrm{ps}$ the value exhibits little change. In the case of $\mathrm{O}-\mathrm{Si}-\mathrm{O}$, we observe that the average intra-tetrahedral bond angle is not affected by the cooling rate. This suggests that the overall tetrahedral structure in the studied bioglass is not significantly affected or distorted by high cooling rates.

Figure 6 shows the inter-tetrahedral BADs. Similar to the intra-tetrahedral BADs, we observe that the SiO-Si BAD exhibits little variation with different cooling rates. Interestingly, we observe that the peak intensity of BAD for Si-O-P decreases and the distribution becomes broader with decreasing cooling rates. This suggests an increased degree of angular disorder within the Si-O-P bridges with decreasing cooling rate.
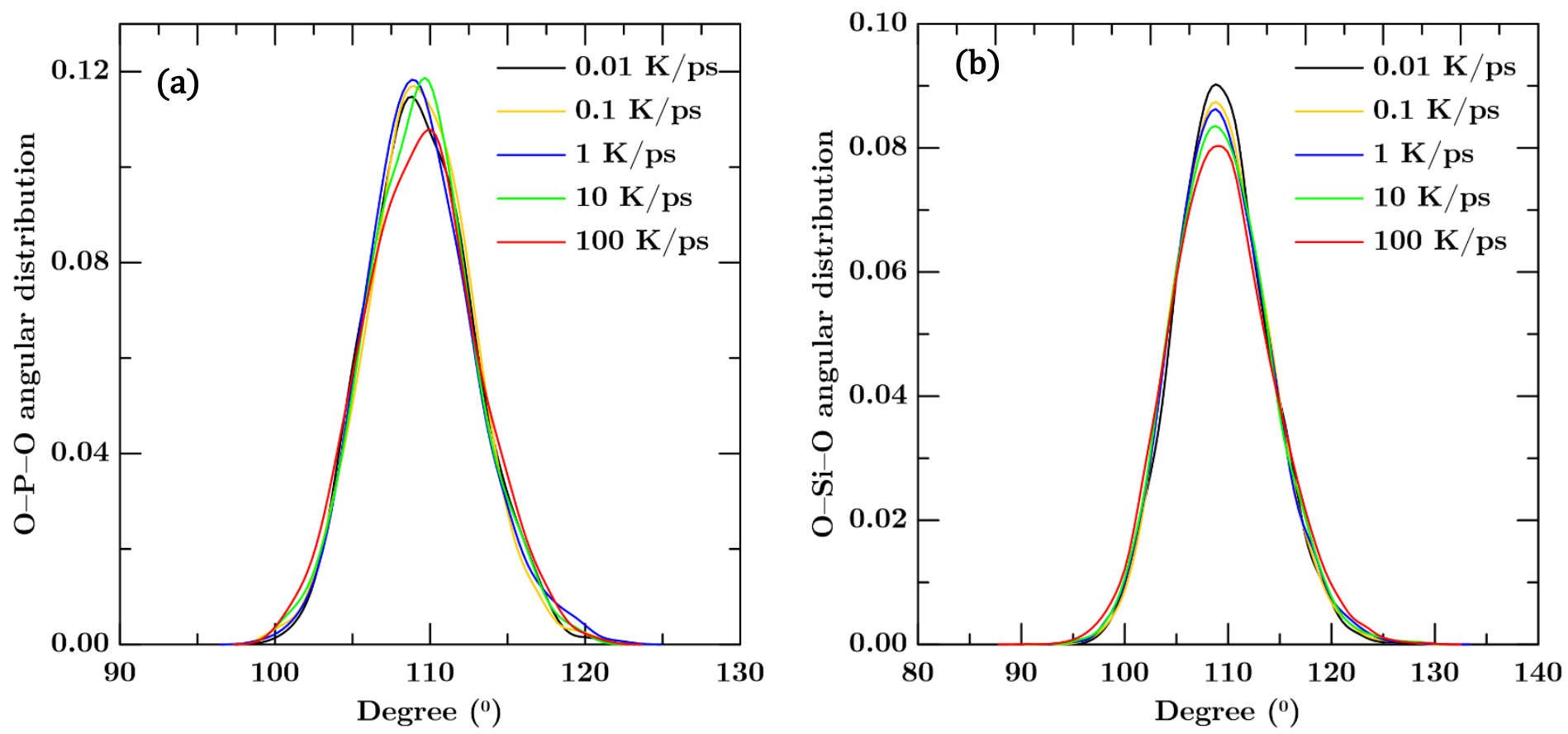

Figure 5. Intra-tetrahedral (a) $\mathrm{O}-\mathrm{P}-\mathrm{O}$ bond and (b) $\mathrm{O}-\mathrm{Si}-\mathrm{O}$ bond angle distribution (BAD) in the glassy state computed by MD simulations for selected cooling rates. 

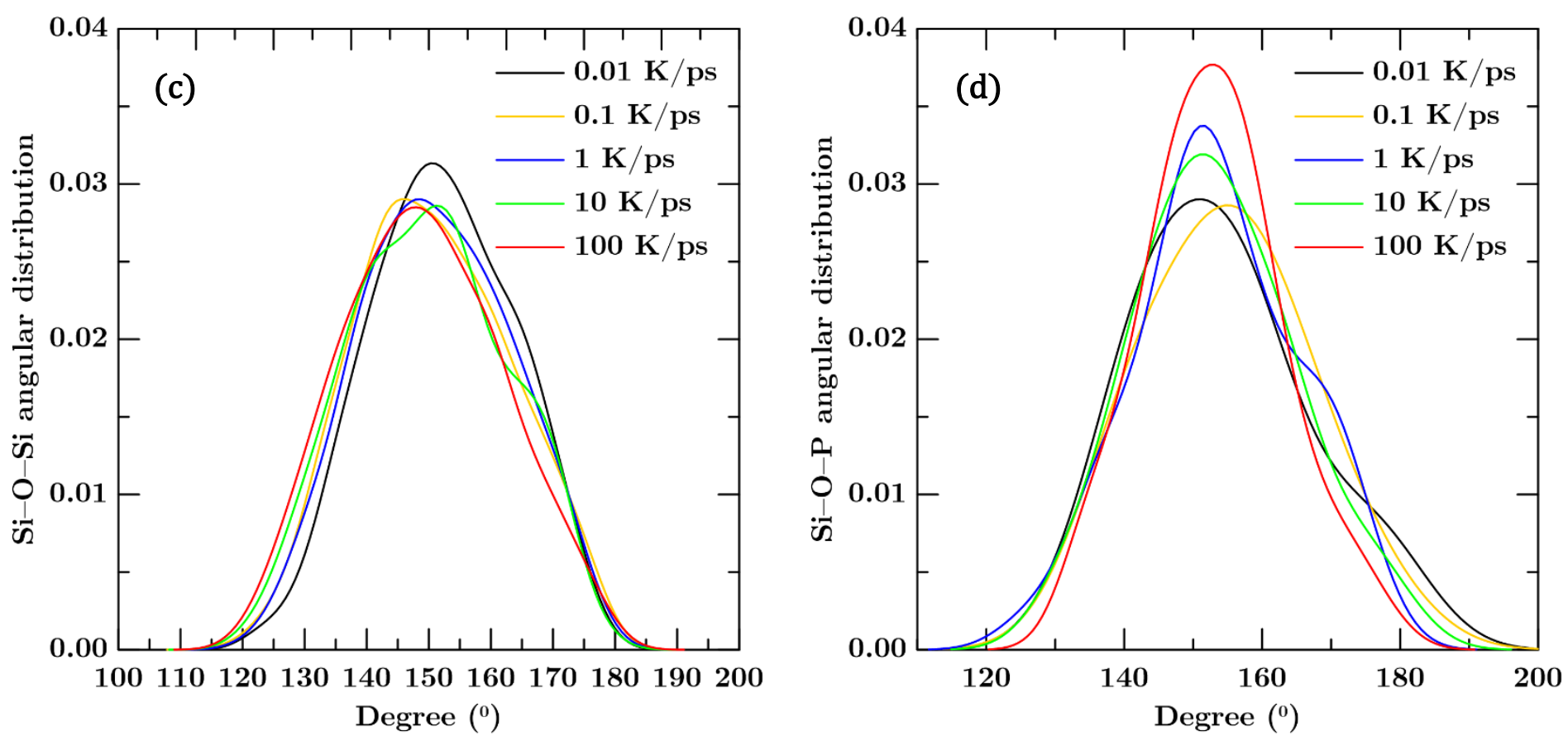

Figure 6. Inter-tetrahedral (a) $\mathrm{Si}-\mathrm{O}-\mathrm{Si}$ and (b) $\mathrm{Si}-\mathrm{O}-\mathrm{P}$ bond angle distribution (BAD) in the glassy state computed by MD simulations for selected cooling rates.

\section{(iii) Network connectivity}

To analyze the effect of the cooling rate on the silicate network connectivity, we quantify the topology of the oxygen atoms. In oxide glasses, the network formers such as Si or P atoms form tetrahedra, which are connected by some $\mathrm{BO}$ atoms through $\mathrm{X}-\mathrm{O}-\mathrm{Y}$ bridges ( $\mathrm{X}$ or $\mathrm{Y}=\mathrm{Si}$ or $\mathrm{P}$ ), thereby increasing the connectivity (or rigidity) of the silicate network. In contrast, network modifiers such as $\mathrm{Na}$ or $\mathrm{Ca}$ atoms tend to depolymerize the network by breaking the $\mathrm{X}-\mathrm{O}-\mathrm{Y}$ bridges leading to the formation of $\mathrm{X}-\mathrm{O}-\mathrm{Na} / \mathrm{Ca}$ bonds. These oxygen atoms are termed as non-bridging oxygen (NBO) atoms. Note that the addition of an $\mathrm{Na}_{2} \mathrm{O}$ species results in the formation of two NBOs through the formation of two $\mathrm{Si}^{-} \mathrm{O}^{-} \mathrm{Na}^{+}$bonds. Similarly, the addition of a $\mathrm{CaO}$ species result in the formation of two NBOs through the formation of a $\mathrm{Si}-\mathrm{O}^{-} \mathrm{Ca}^{2+}-\mathrm{O}^{-}-\mathrm{Si}[32]$.

To this extent, we compute the fraction of Si-O-P and $\mathrm{Si}-\mathrm{O}-\mathrm{Si}$ linkages and compare them with the predictions from a random network model.[62] According to this model, each network former (that is, Si or P) can pick its neighbor with equal probability from the available atoms, irrespective of the atom type. Thus, the fractions of $\mathrm{Si}-\mathrm{O}-\mathrm{Si}, \mathrm{Si}-\mathrm{O}-\mathrm{P}, \mathrm{P}-\mathrm{O}-\mathrm{P}$, angles are given by

$$
\begin{aligned}
f_{S i-O-S i} & =\frac{4 N_{S i} \times 4 N_{S i}}{\left(4 N_{S i}+4 N_{S i}\right)^{2}} \\
f_{S i-O-P} & =\frac{4 N_{S i} \times 3 N_{P}}{\left(4 N_{S i}+3 N_{P}\right)^{2}} \\
f_{P-O-P} & =\frac{3 N_{P} \times 3 N_{P}}{\left(3 N_{P}+3 N_{P}\right)^{2}}
\end{aligned}
$$


where $N_{S i}$ and $N_{P}$ are the numbers of Si and P atoms, respectively.

Figure 7 shows the fraction of the $\mathrm{Si}-\mathrm{O}-\mathrm{Si}$ and $\mathrm{Si}-\mathrm{O}-\mathrm{P}$ linkages predicted from the random model compared with the results from MD simulations for different cooling rates. We observe that, overall, the glasses exhibit a chemically-ordered structure that deviates from the predictions of the random modelwherein the computed fractions of $\mathrm{Si}-\mathrm{O}-\mathrm{P}$ and $\mathrm{Si}-\mathrm{O}-\mathrm{Si}$ linkages are lower and higher than those obtained from a random distribution, respectively. Note that the $\mathrm{P}-\mathrm{O}-\mathrm{P}$ linkages are absent in structures generated from MD simulations, in agreement with experimental observations. As the cooling rate decreases, the deviation from the random model increases and the glass exhibits an enhanced chemically-ordered structure. Further, the fraction of Si-O-P linkages decrease (and Si-O-Si linkages increase) with decreasing cooling rate. Overall, these results suggest that the phosphate species exhibit a tendency to form isolated orthophosphate tetrahedra in bioglass - the manifestation of which is closely linked to the cooling rate used to prepare the glass.

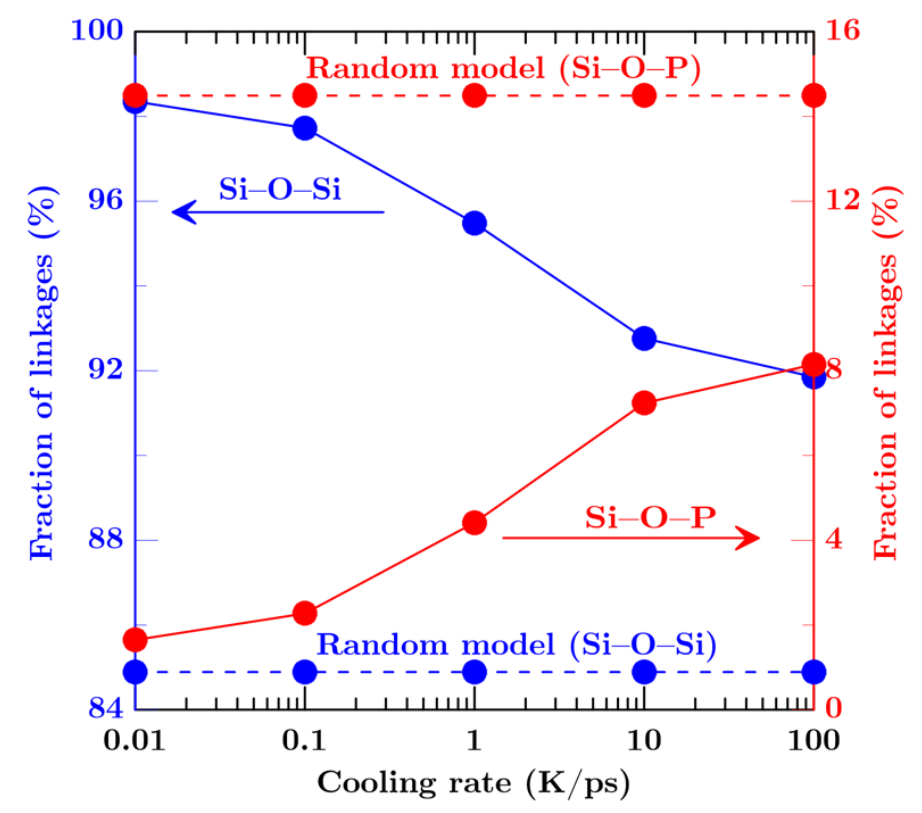

Figure 7. Fraction of $\mathrm{Si}-\mathrm{O}-\mathrm{Si}$ and $\mathrm{Si}-\mathrm{O}-\mathrm{P}$ linkages predicted from the random model compared with the results from MD simulations for different cooling rates. 


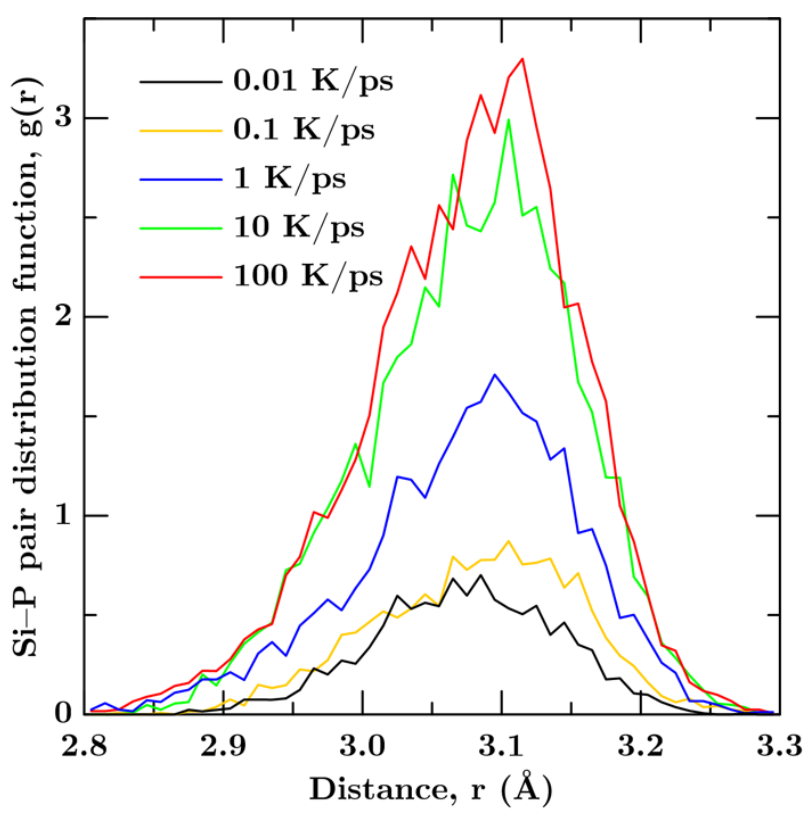

Figure 8. Si-P partial pair distribution functions in the glassy state for selected cooling rates computed by MD simulations.

To confirm the increased affinity of P toward NBOs, we compute the Si-P partial PDF (see Fig. 8). Upon decreasing cooling rate, the intensity of the Si-P peak diminishes, and the peak becomes notably less intense. Note that this is in contrast to the other partial PDFs (Figs. 3 and 4), which exhibit sharper peaks with decreasing cooling rates. This confirms that the tendency of $\mathrm{P}$ atoms to form isolated orthophosphate species, which is magnified upon decreasing cooling rate, i.e., when the glass reaches a more stable state. In other words, for a system obtained with reasonably low cooling rates, such as the experimental glasses, there is a tendency to avoid the formation of $\mathrm{X}-\mathrm{O}-\mathrm{P}$ bridges. Instead, the phosphate tetrahedra tends to remain as isolated $\left[\mathrm{PO}_{4}\right]^{3-}$ species with four NBOs. This observation is consistent with previous experimental studies, which report a low fraction of $Q^{1} P$ species.[26,27]

\section{(iv) $\mathrm{Q}^{\mathrm{n}}$ distribution}

To study the connectivity of the tetrahedral network further, we analyze the $Q^{n}$ distribution (see Methodology section) both computationally and from solid-state NMR measurements on a bioglass sample. The fitting parameters used herein for both the ${ }^{31} \mathrm{P}$ and ${ }^{29} \mathrm{Si}$ MAS NMR data, as well as the obtained $Q^{n}$ distributions, are presented in Tables 1 and 2.

Table 1. Parameters used for deconvolution of ${ }^{31} \mathrm{P}$ MAS NMR spectra of 4555 bioglass. The $Q_{p}{ }^{n}$ populations (atom \%) include intensity from the spinning sidebands.

\begin{tabular}{|c|c|c|c|l|c|c|c|c|}
\hline $\mathbf{Q}_{\mathbf{p}}{ }^{\mathbf{( \% )}}$ & $\begin{array}{c}\text { Pos } \\
(\mathrm{ppm})\end{array}$ & $\begin{array}{c}\text { Width } \\
(\mathrm{ppm})\end{array}$ & $\begin{array}{c}\text { Gaus/Lor } \\
(\%)\end{array}$ & $\mathbf{Q}_{\mathbf{p}}{ }^{\mathbf{1}} \mathbf{( \% )}$ & $\begin{array}{c}\text { Pos } \\
(\mathrm{ppm})\end{array}$ & $\begin{array}{c}\text { Width } \\
(\mathrm{ppm})\end{array}$ & $\begin{array}{c}\text { Gaus/Lor } \\
(\%)\end{array}$ \\
\hline $\mathbf{9 7}$ & 8.9 & 8.0 & 0.8 & & $\mathbf{3}$ & 2.0 & 8.7 & 1 \\
\hline
\end{tabular}




\begin{tabular}{|c|c|c|c|c|c|c|c|c|}
\hline$Q_{s i}{ }^{1}(\%)$ & $\begin{array}{c}\text { Pos } \\
\text { (ppm) }\end{array}$ & $\begin{array}{l}\text { Width } \\
\text { (ppm) }\end{array}$ & $Q_{s i}{ }^{2}(\%)$ & $\begin{array}{c}\text { Pos } \\
\text { (ppm) }\end{array}$ & $\begin{array}{l}\text { Width } \\
\text { (ppm) }\end{array}$ & $Q_{s i}^{3}(\%)$ & $\begin{array}{c}\text { Pos } \\
\text { (ppm) }\end{array}$ & $\begin{array}{l}\text { Width } \\
\text { (ppm) }\end{array}$ \\
\hline 6 & -69.8 & 7.8 & 77 & -78.4 & 10.45 & 17 & -86.3 & 11.8 \\
\hline
\end{tabular}

Figures $9(\mathrm{a})$ and $(\mathrm{b})$ show the ${ }^{29} \mathrm{Si}$ and ${ }^{31} \mathrm{P}$ MAS NMR with deconvolution into respective $\mathrm{Q}^{\mathrm{n}}$ species. From ${ }^{29} \mathrm{Si}$ MAS NMR, we observe that the fraction of $\mathrm{Qsi}^{2}$ units is approximately $76 \%$, while that of $\mathrm{Qsi}^{1}{ }^{1}$ and $\mathrm{Qsi}^{3}$ are approximately $5 \%$ and $17 \%$, respectively (Table 2 ). The present experimental results from ${ }^{29}$ Si MASNMR are in agreement with previous studies by Yu et al.[29] and Pedone et al.[20]. These results suggest that the silicate network primarily consists of $\mathrm{Qsi}^{2}$ tetrahedra, with some degree of cross-linking provided by $Q_{s i}{ }^{3}$ species. From ${ }^{31} \mathrm{P}$ MAS-NMR, we observe that $P$ atoms exists only as $Q^{0}$ and $Q^{1}$ units, wherein the fraction of $Q^{0}$ atoms is around $97 \%$ (Table 1). The residuals associated with the fit is provided in the supplementary material. This confirms that the $\mathrm{P}$ atoms prefer to exist mostly as isolated $\mathrm{Q}^{0}$ orthophosphate ions in the bioglass system, although non-trivial amounts (3\%) of $\mathrm{Q}^{1}$ may exist. This observation is in agreement with Yu et al. [29], where 4.1\% of $Q_{p}{ }^{1}$ were observed. Further, the chemical shifts for $Q_{p}{ }^{0}$ and $Q_{p}{ }^{1}$ observed by $Y u$ et al. are 8.7 and 0.4 ppm, respectively, which are comparable to the chemical shifts of 8.9 and $2.0 \mathrm{ppm}$, respectively, observed in the present study. It should be noted that $Q_{P}^{0}$ and $Q_{P}^{1}$ exhibit different chemical shift anisotropy (CSA). Since, CSA is highly sensitive to symmetry, $Q_{P}{ }^{0}$ is expected to exhibit a smaller CSA. Based on an accurate fit ${ }^{31} P$ MAS NMR spectrum utilizing all spinning sidebands, we estimate the CSA of $Q_{p}^{0}$ and $Q_{p}{ }^{1}$ as 56 and 124 ppm, respectively. Overall, the present work conclusively provides the evidence for the existence of $Q_{p}{ }^{1}$ species, although in minor fractions of around $3 \%$.
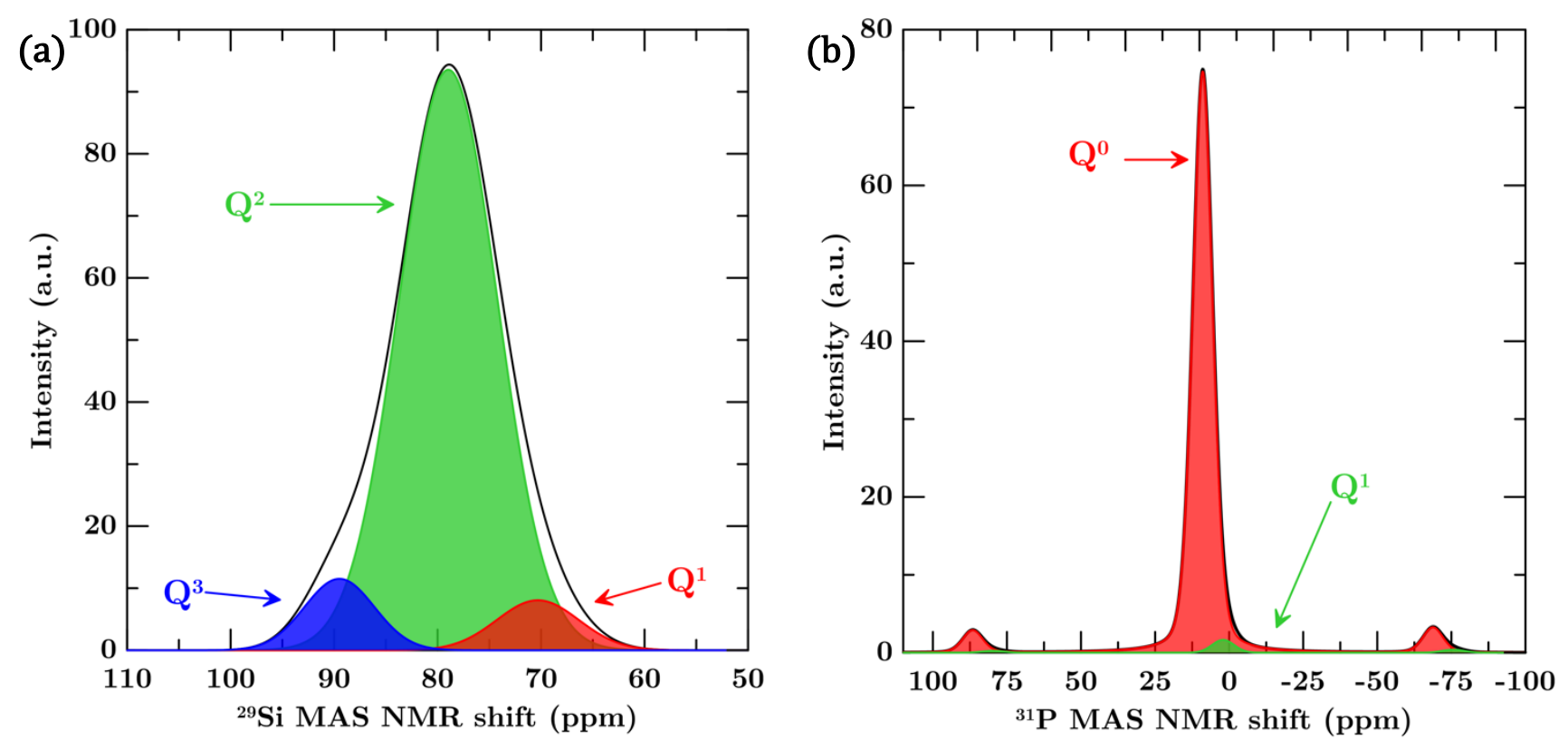

Figure 9. (a) ${ }^{29} \mathrm{Si}$ MAS NMR spectrum of bioglass with deconvolution into $Q^{1}, Q^{2}$ and $Q^{3}$ silicate groups and (b) ${ }^{31} \mathrm{P}$ MAS NMR spectrum of bioglass with deconvolution into $Q^{0}$ and $Q^{1}$ phosphate groups. 
Now, we compare the experimental results with the $Q^{n}$ distributions obtained from MD simulations. Figures 10(a) and (b) shows the $Q^{n}$ distributions of the tetrahedral species in the system formed by $\mathrm{Si}$ and $\mathrm{P}$ atoms, respectively. Note that, in addition to the experimental and simulation results from the present study, experimental results from Yu et al.[29] and Pedone et al.[20] and simulation results from Pedone et al.[20] are included for comparison. In the case of Si, MD simulations results suggest that a large fraction (around 40-45\%) of the glass network comprises of $\mathrm{Qsi}^{2}$ tetrahedra, at the expense of $\mathrm{Qsi}_{\mathrm{si}}{ }^{3}$ or $\mathrm{Q}_{\mathrm{si}}{ }^{4}$ tetrahedra. Further, we observe that the fraction of $\mathrm{Q}_{\mathrm{si}}{ }^{0}, \mathrm{Q}_{\mathrm{si}}{ }^{1}$, and $\mathrm{Q}_{\mathrm{si}}{ }^{4}$ units decreases with decreasing cooling rate. Now, the simulated values are extrapolated to experimental cooling rates by fitting a line in the least squared sense (see Fig. 10(a)). The extrapolated values are then compared to experimental results as shown in Fig. 10(a). We observe that the trend in $\mathrm{Q}^{\text {n }}$ distribution of $\mathrm{Si}$ is wellpredicted by the MD simulations. In particular, the fraction of $Q^{3}$ units exhibit an excellent match when extrapolated. However, $\mathrm{Q}^{2}$ units are slightly underpredicted while the $\mathrm{Q}^{1}$ units are overpredicted by the extrapolation from MD simulations. Overall, we observe that the trend predicted by MD simulations exhibits a good match with experiments for the $Q_{s i}^{n}$ units of Si atoms.

In the case of the $P$ atoms, we observe that the structure exhibits only $Q^{0}, Q^{1}$, and $Q^{2}$ units, even at the highest cooling rates (see Fig. 10(b)). Upon decreasing cooling rate, $\mathrm{Q}^{2}$ units decrease quickly and converge to zero, even within the range of cooling rates accessible by MD simulations. Further, the fractions $Q^{0}$ and $Q^{1}$ units monotonically increase and decrease with decreasing cooling rate, respectively. This confirms that, as the cooling rate decreases, $\mathrm{P}$ atoms increasingly tend to form some isolated $\left[\mathrm{PO}_{4}\right]^{3-}$ tetrahedral units. These values are then compared to the experimental results obtained using ${ }^{31} \mathrm{P}$ MASNMR by extrapolating the MD results in a least squared sense. Interestingly, we observe that the extrapolation of MD simulation suggests the existence of $\mathrm{Q}^{0}$ species only, not accounting for the minor $Q_{P}{ }^{1}$ species suggested by the experiments. However, this could be due to the low amount of $P$ atoms present in the bioglass system, making an accurate determination of the $Q^{1}$ units challenging. Extending MD simulations to lower cooling rates or increasing the size of the system may provide deeper insights into the possible existence of $Q_{p}{ }^{1}$ units. Overall, we observe that the effect of the cooling rate on the $Q^{n}$ distributions can be reasonably captured when MD simulations are extrapolated to experimental timescales, and furthermore, the experimentally-determined network structure of bioglass is consistent with previous studies of bioglasses. 

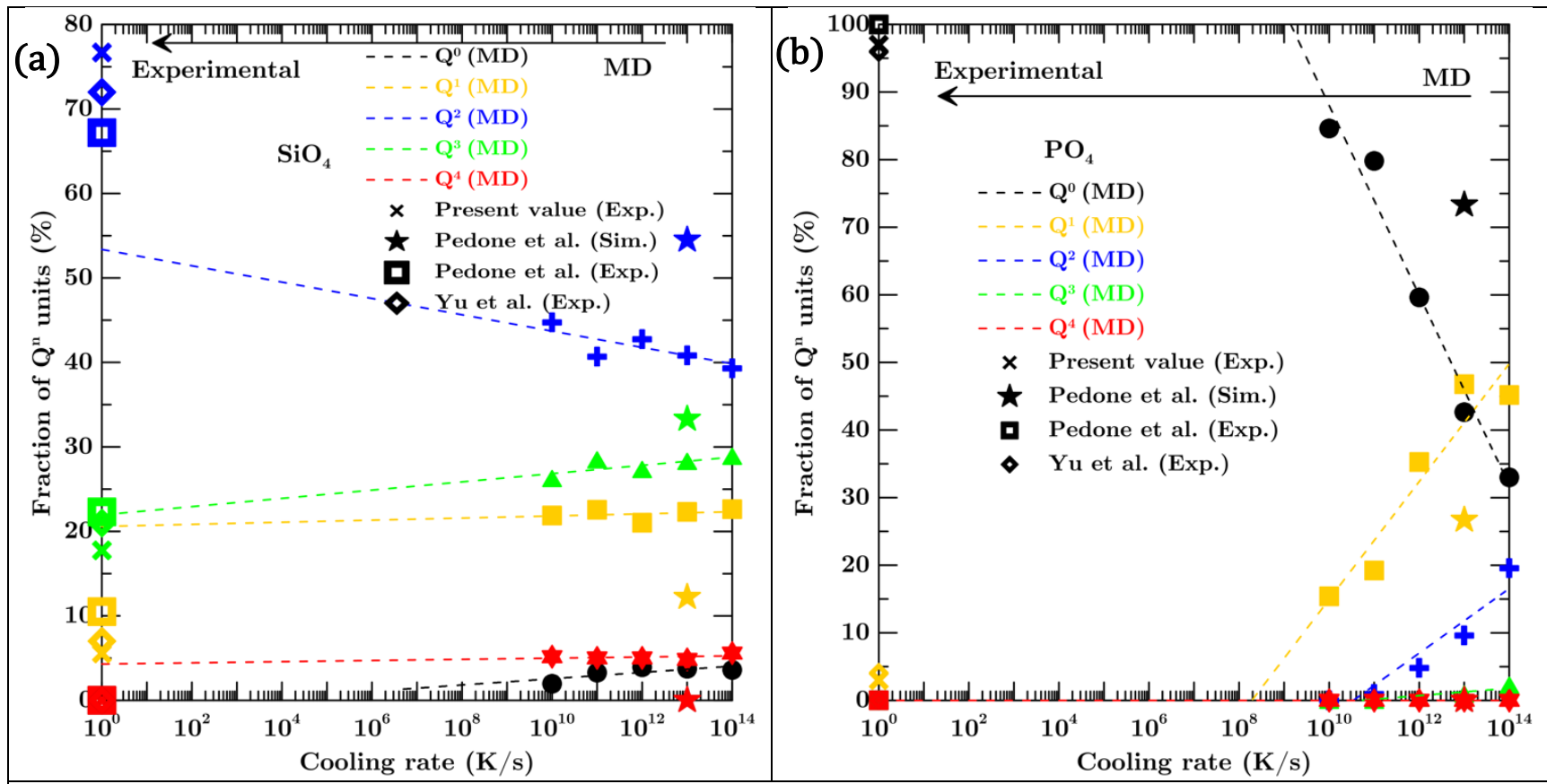

Figure 10. $Q^{n}$ distribution of (a) Si and (b) $P$ tetrahedra in $45 S 5$ Bioglass computed by MD simulations for five different cooling rates. Experimental results obtained by MAS-NMR and from Yu et al.[29] and Pedone et al.[20] and simulation results from Pedone et al.[20] are added for comparison. The lines are fitted using the MD simulation data in a least-squared sense.

\section{(v) Medium-range order}

While the PDF is useful for analyzing the structure correlations at smaller distances $(\leq 3 \AA)$, the structure factor represents the correlations at larger distances (between $3 \AA$ to $10 \AA$ ). In particular, the first sharp diffraction peak (FSDP) captures the extent of correlation in the medium-range structure of glasses.[42,63-66] The full width at half-maximum (FWHM) of the FSDP is inversely linked to a mediumrange coherence length $L$ as, $L=7.7 / \mathrm{FWHM},[42,63-66]$ which captures the medium-range correlation distance among fluctuations of atomic density. Figure 12(a) shows the neutron structure factors computed by MD simulations with different cooling rates. We observe that the structure factor at larger values of wave vector exhibits little differences. However, FSDP becomes sharper with increasing peak intensity for decreasing cooling rates. This indicates that, in turn, the coherence length in the mediumrange order increases upon decreasing cooling rate. To confirm this, we plot the FWHM and coherence length in Fig. 12(b). We observe that, upon decreasing cooling rate, FWHM decreases and the coherence length increases (see Fig. 12(b)), which denotes an increased degree of order within the medium-range structure of the glass. This echoes the fact that, due to the tendency $P$ atoms to exist as isolated species, the network presents an increasingly chemically-ordered structure upon decreasing cooling rate-in agreement with the experimental and simulation data on $Q^{n}$ as well. 

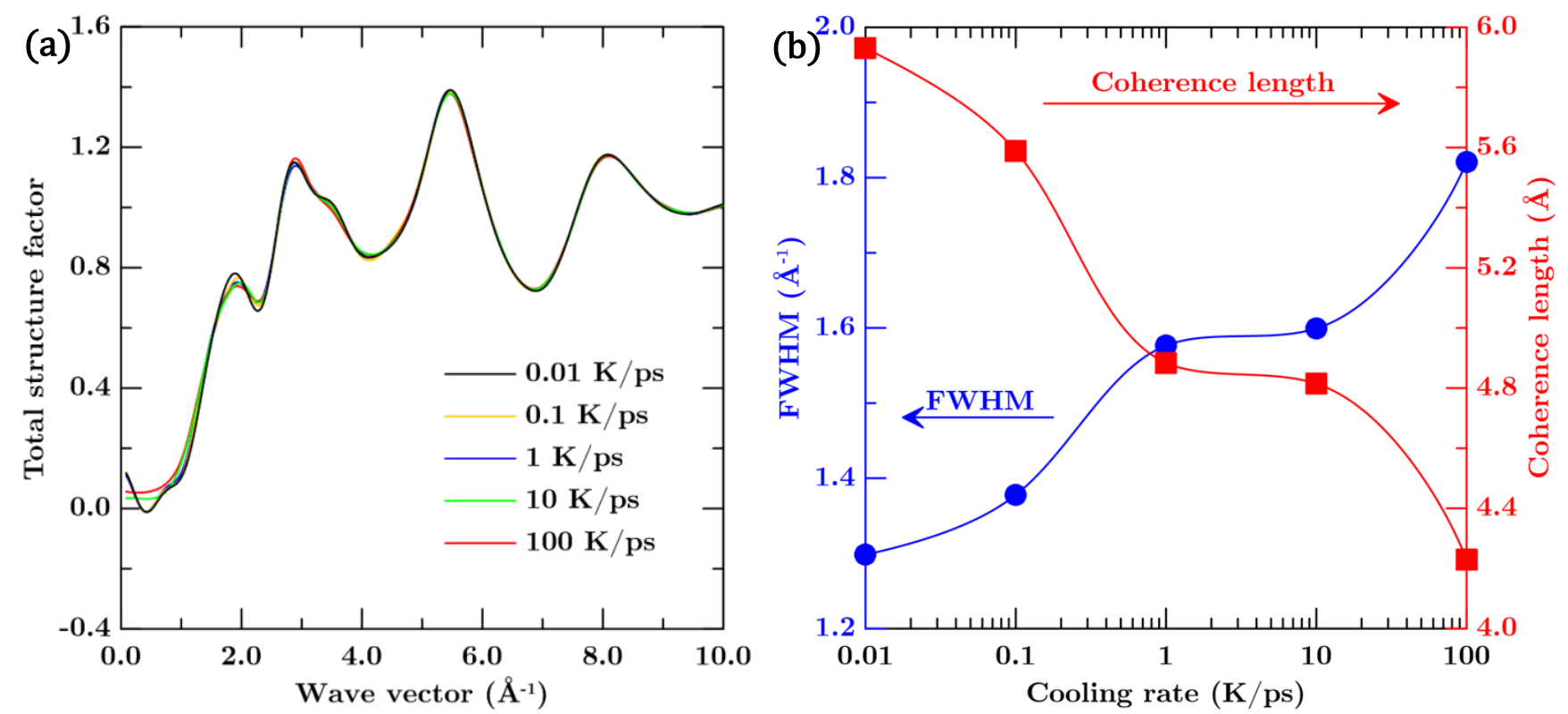

Figure 12. (a) Computed total neutron structure factors in the glassy state for selected cooling rates. (b) Full width at half maxima (FWHM) of the total structure factor as a function of cooling rate (left yaxis) and the corresponding coherence length (right y-axis). The line is a guide for the eye.

Finally, we analyze the effect of the cooling rate on the ring size distribution within the network computed by MD simulations (see Methodology section). As shown in Fig. 13, we find that, in contrast to the short-range order, the ring distribution is largely affected by the cooling rate. First, we observe that there are no two-membered rings, which confirms the absence of any edge-sharing tetrahedra. Further, there are no small three-membered rings for all but the highest cooling rate of $100 \mathrm{~K} / \mathrm{ps}$. This arises from the fact that, at high cooling rate, atoms may get frozen in unstable states due to the limited time available for structural rearrangements. With decreasing cooling rate, we observe that larger rings are preferred with a maximum fraction observed for five-membered rings, that is, rings having five Si atoms. In particular, a sharp peak at a ring size of 5 is observed for the cooling rate of $0.01 \mathrm{~K} / \mathrm{ps}$, which shows that almost $50 \%$ of the rings have a size of five Si atoms. This is in contrast with glassy silica, wherein a ring size of six is preferred.[42,43,67] Finally, we observe that all the rings are formed by Si and $\mathrm{O}$ atoms, thereby confirming that the $\mathrm{P}$ atoms do not participate in the ring formation. This is consistent with the fact that $P$ atoms mostly form isolated $Q^{0}$ and terminating $Q^{1}$ units and, hence, are unable to participate in these ring structures. Overall, we conclude that the medium range structure of the bioglass is significantly affected by the cooling rate. 


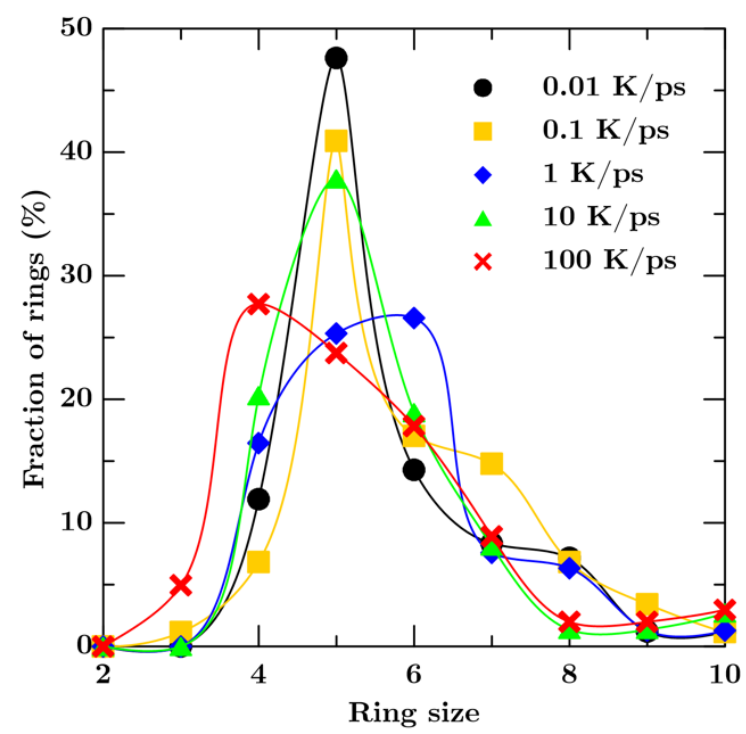

Figure 13. Ring distribution for the bioglass at various cooling rates computed by MD simulations.

The present results suggest a holistic picture reconciling earlier experimental and MD-simulation-based studies. While the short-range structure of bioglass hardly depends on the cooling rate, the medium range structure is significantly affected. In particular, our results confirm that $P$ atoms exhibit an increased affinity to NBOs, thereby leading to the gradual disappearance of Si-O-P bridges at lower cooling rates. In other words, lower cooling rate results in an increase in the concentration of isolated orthophosphate anions, which associate with the $\mathrm{Ca}$ and $\mathrm{Na}$ cations present in the structure. As such, the extrapolation of MD results toward lower experimental cooling rates would result in: (i) a significant decrease in the fraction of Si-O-P bridges, (ii) the absence of any $\mathrm{Q}_{\mathrm{p}}{ }^{2}$ species, as observed in our NMR measurements, and (iii) an increased propensity for $\mathrm{P}$ atoms to exist as isolated orthophosphate ions. Indeed, it is well known that the high cooling rates used in MD simulations tend to overestimate the degree of randomness in computed $Q^{n}$ distributions. Overall, the present study emphasizes the importance of using a cooling rate study in MD simulations covering different orders of magnitude to reconcile experimental and simulation structural studies.

From a more general perspective, these results can have significant ramifications in the understanding of the structure and bioactivity of bioglass. It is well known that bioactivity is closely related to the leaching of ions from the glass during the dissolution process.[68] This occurs through the breakage of interatomic bonds present in the glass structure. As such, the absence of a large number of Si-O-P bridges, which were otherwise postulated by previous MD studies, suggests that isolated phosphate ions may exhibit an increased mobility and, hence, can easily leach out from the glass. This is further confirmed by the NMR experiments wherein approximately $97 \%$ of $P$ atoms are shown to exist as $Q^{0}$ units. This may in turn contribute to the bioactive behavior of the glass. However, confirming this hypothesis would require future experimental studies on the degree of congruency in the leaching rates of Si and P species in bioglass.

\section{Conclusions}

The present study reveals the importance of the cooling rate in governing the structure of bioactive glasses. In particular, we show that the medium-range order structure is largely controlled by the 
thermal history of the glass. Interestingly, we observe an increased affinity of $\mathrm{P}$ atoms toward NBOs leading to a significant decrease in the fraction of Si-O-P bridges upon lower cooling rates. This observation, along with the propensity for $\mathrm{P}$ atoms to form isolated tetrahedra, confirms the earlier experimental findings that phosphorus atoms primarily exist as isolated orthophosphate ions in bioglass, although minor quantities of $Q_{p}^{1}$ may also exist. More importantly, we show that, through a detailed analysis of cooling rate simulations covering several order magnitudes, the experimental results can be reasonably extrapolated using MD simulations. This, in turn, can provide a handshake between MD simulation and experiments, which is otherwise challenging due to the extremely high cooling rates involved in MD simulations.

\section{Acknowledgement}

NMAK acknowledge the financial support for this research provided by the Department of Science and Technology, India under the INSPIRE faculty scheme (DST/INSPIRE/04/2016/002774) and DST SERB Early Career Award (ECR/2018/002228). Authors thank Corning Inc. for the experimental resources and IIT Delhi HPC facility for providing the computational and storage resources. MB acknowledges funding from the National Science Foundation under Grants No. 1562066, 1762292, and 1826420.

\section{References:}

[1] L.L. Hench, Ö. Andersson, Bioactive glasses, in: An Introduction to Bioceramics, WORLD SCIENTIFIC, 1993: pp. 41-62. http://www.worldscientific.com/doi/abs/10.1142/9789814317351_0003.

[2] M.N. Rahaman, D.E. Day, B. Sonny Bal, Q. Fu, S.B. Jung, L.F. Bonewald, A.P. Tomsia, Bioactive glass in tissue engineering, Acta Biomaterialia. 7 (2011) 2355-2373. doi:10.1016/j.actbio.2011.03.016.

[3] N.C. Lindfors, P. Hyvönen, M. Nyyssönen, M. Kirjavainen, J. Kankare, E. Gullichsen, J. Salo, Bioactive glass S53P4 as bone graft substitute in treatment of osteomyelitis, Bone. 47 (2010) 212-218. doi:10.1016/j.bone.2010.05.030.

[4] P. Stoor, E. Söderling, J.I. Salonen, Antibacterial effects of a bioactive glass paste on oral microorganisms, Acta Odontologica Scandinavica. 56 (1998) 161-165. doi:10.1080/000163598422901.

[5] S. Srinivasan, R. Jayasree, K.P. Chennazhi, S.V. Nair, R. Jayakumar, Biocompatible alginate/nano bioactive glass ceramic composite scaffolds for periodontal tissue regeneration, Carbohydrate Polymers. 87 (2012) 274-283. doi:10.1016/j.carbpol.2011.07.058.

[6] J. Kent Leach, D. Kaigler, Z. Wang, P.H. Krebsbach, D.J. Mooney, Coating of VEGF-releasing scaffolds with bioactive glass for angiogenesis and bone regeneration, Biomaterials. 27 (2006) 3249-3255. doi:10.1016/j.biomaterials.2006.01.033.

[7] J.M. Gomez-Vega, E. Saiz, A.P. Tomsia, G.W. Marshall, S.J. Marshall, Bioactive glass coatings with hydroxyapatite and Bioglass ${ }^{\circledR}$ particles on Ti-based implants. 1. Processing, Biomaterials. 21 (2000) 105-111. doi:10.1016/S0142-9612(99)00131-3.

[8] Y. Xiang, J. Du, Effect of Strontium Substitution on the Structure of $45 S 5$ Bioglasses, Chemistry of Materials. 23 (2011) 2703-2717. doi:10.1021/cm102889q.

[9] A. Tilocca, A.N. Cormack, N.H. de Leeuw, The Structure of Bioactive Silicate Glasses: New Insight from Molecular Dynamics Simulations, Chemistry of Materials. 19 (2007) 95-103. doi:10.1021/cm061631g.

[10] A. Tilocca, Cooling rate and size effects on the medium-range structure of multicomponent oxide glasses simulated by molecular dynamics, J. Chem. Phys. 139 (2013) 114501. doi:10.1063/1.4821150.

[11] A. Tilocca, Short- and medium-range structure of multicomponent bioactive glasses and melts: An assessment of the performances of shell-model and rigid-ion potentials, J. Chem. Phys. 129 (2008) 084504. doi:10.1063/1.2972146. 
[12] A. Tilocca, Structure and dynamics of bioactive phosphosilicate glasses and melts from ab initio molecular dynamics simulations, Phys. Rev. B. 76 (2007) 224202. doi:10.1103/PhysRevB.76.224202.

[13] A. Tilocca, A.N. Cormack, Structural Effects of Phosphorus Inclusion in Bioactive Silicate Glasses, J. Phys. Chem. B. 111 (2007) 14256-14264. doi:10.1021/jp075677o.

[14] R. Mathew, B. Stevensson, A. Tilocca, M. Edén, Toward a Rational Design of Bioactive Glasses with Optimal Structural Features: Composition-Structure Correlations Unveiled by Solid-State NMR and MD Simulations, J. Phys. Chem. B. 118 (2014) 833-844. doi:10.1021/jp409652k.

[15] J. Du, Y. Xiang, Effect of strontium substitution on the structure, ionic diffusion and dynamic properties of 45S5 Bioactive glasses, Journal of Non-Crystalline Solids. 358 (2012) 1059-1071. doi:10.1016/j.jnoncrysol.2011.12.114.

[16] K. Fujikura, N. Karpukhina, T. Kasuga, D. S. Brauer, R. G. Hill, R. V. Law, Influence of strontium substitution on structure and crystallisation of Bioglass ${ }^{\circledR}$ 45S5, Journal of Materials Chemistry. 22 (2012) 7395-7402. doi:10.1039/C2JM14674F.

[17] F. Fayon, C. Duée, T. Poumeyrol, M. Allix, D. Massiot, Evidence of Nanometric-Sized Phosphate Clusters in Bioactive Glasses As Revealed by Solid-State 31P NMR, J. Phys. Chem. C. 117 (2013) 2283-2288. doi:10.1021/jp312263j.

[18] R. Mathew, C. Turdean-lonescu, B. Stevensson, I. Izquierdo-Barba, A. García, D. Arcos, M. Vallet-Regí, M. Edén, Direct Probing of the Phosphate-Ion Distribution in Bioactive Silicate Glasses by Solid-State NMR: Evidence for Transitions between Random/Clustered Scenarios, Chem. Mater. 25 (2013) 1877-1885. doi:10.1021/cm400487a.

[19] E. Berardo, A. Pedone, P. Ugliengo, M. Corno, DFT Modeling of 4555 and 77S Soda-Lime Phospho-Silicate Glass Surfaces: Clues on Different Bioactivity Mechanism, Langmuir. 29 (2013) 5749-5759. doi:10.1021/la304795w.

[20] A. Pedone, T. Charpentier, G. Malavasi, M.C. Menziani, New Insights into the Atomic Structure of 4555 Bioglass by Means of Solid-State NMR Spectroscopy and Accurate First-Principles Simulations, Chem. Mater. 22 (2010) 5644-5652. doi:10.1021/cm102089c.

[21] A. Pedone, G. Malavasi, M.C. Menziani, Computational Insight into the Effect of CaO/MgO Substitution on the Structural Properties of Phospho-Silicate Bioactive Glasses, J. Phys. Chem. C. 113 (2009) 15723-15730. doi:10.1021/jp904131t.

[22] M. Corno, A. Pedone, R. Dovesi, P. Ugliengo, B3LYP Simulation of the Full Vibrational Spectrum of 45S5 Bioactive Silicate Glass Compared to v-Silica, Chem. Mater. 20 (2008) 5610-5621. doi:10.1021/cm801164u.

[23] B. Stevensson, R. Mathew, M. Edén, Assessing the Phosphate Distribution in Bioactive Phosphosilicate Glasses by 31P Solid-State NMR and Molecular Dynamics Simulations, J. Phys. Chem. B. 118 (2014) 88638876. doi:10.1021/jp504601c.

[24] Y. Yu, B. Stevensson, M. Edén, Medium-Range Structural Organization of Phosphorus-Bearing Borosilicate Glasses Revealed by Advanced Solid-State NMR Experiments and MD Simulations: Consequences of B/Si Substitutions, J. Phys. Chem. B. 121 (2017) 9737-9752. doi:10.1021/acs.jpcb.7b06654.

[25] B. Stevensson, Y. Yu, M. Edén, Structure-composition trends in multicomponent borosilicate-based glasses deduced from molecular dynamics simulations with improved $\mathrm{B}-\mathrm{O}$ and $\mathrm{P}-\mathrm{O}$ force fields, Physical Chemistry Chemical Physics. 20 (2018) 8192-8209. doi:10.1039/C7CP08593A.

[26] V. FitzGerald, D.M. Pickup, D. Greenspan, G. Sarkar, J.J. Fitzgerald, K.M. Wetherall, R.M. Moss, J.R. Jones, R.J. Newport, A Neutron and X-Ray Diffraction Study of Bioglass ${ }^{\circledR}$ with Reverse Monte Carlo Modelling, Advanced Functional Materials. 17 (2007) 3746-3753. doi:10.1002/adfm.200700433.

[27] M. Cerruti, C.L. Bianchi, F. Bonino, A. Damin, A. Perardi, C. Morterra, Surface Modifications of Bioglass Immersed in TRIS-Buffered Solution. A Multitechnical Spectroscopic Study, The Journal of Physical Chemistry B. 109 (2005) 14496-14505. doi:10.1021/jp050705t.

[28] I. Elgayar, A.E. Aliev, A.R. Boccaccini, R.G. Hill, Structural analysis of bioactive glasses, Journal of NonCrystalline Solids. 351 (2005) 173-183. doi:10.1016/j.jnoncrysol.2004.07.067. 
[29] Y. Yu, M. Edén, Structure-composition relationships of bioactive borophosphosilicate glasses probed by multinuclear ${ }^{11} \mathrm{~B},{ }^{29} \mathrm{Si}$, and $31 \mathrm{P}$ solid state NMR, RSC Advances. 6 (2016) 101288-101303. doi:10.1039/C6RA15275A.

[30] M.D. O'Donnell, S.J. Watts, R.V. Law, R.G. Hill, Effect of P2O5 content in two series of soda lime phosphosilicate glasses on structure and properties - Part I: NMR, Journal of Non-Crystalline Solids. 354 (2008) 3554-3560. doi:10.1016/j.jnoncrysol.2008.03.034.

[31] J. Wilson, G.H. Pigott, F.J. Schoen, L.L. Hench, Toxicology and biocompatibility of bioglasses, Journal of Biomedical Materials Research. 15 (1981) 805-817. doi:10.1002/jbm.820150605.

[32] A.K. Varshneya, Fundamentals of Inorganic Glasses, Elsevier, 2013.

[33] E.D. Zanotto, J.C. Mauro, The glassy state of matter: Its definition and ultimate fate, Journal of NonCrystalline Solids. (n.d.). doi:10.1016/j.jnoncrysol.2017.05.019.

[34] R. Ravinder, R. Kumar, M. Agarwal, N.M.A. Krishnan, Evidence of a two-dimensional glass transition in graphene: Insights from molecular simulations, Scientific Reports. 9 (2019) 4517. doi:10.1038/s41598-01941231-z.

[35] H. Shintani, H. Tanaka, Frustration on the way to crystallization in glass, Nature Physics. 2 (2006) 200-206. doi:10.1038/nphys235.

[36] K. Vollmayr, W. Kob, K. Binder, How do the properties of a glass depend on the cooling rate? A computer simulation study of a Lennard-Jones system, J. Chem. Phys. 105 (1996) 4714-4728. doi:10.1063/1.472326.

[37] K. Vollmayr, W. Kob, K. Binder, Cooling-rate effects in amorphous silica: A computer-simulation study, Physical Review B. 54 (1996) 15808-15827. doi:10.1103/PhysRevB.54.15808.

[38] J.M.D. Lane, Cooling rate and stress relaxation in silica melts and glasses via microsecond molecular dynamics, Physical Review E. 92 (2015) 012320. doi:10.1103/PhysRevE.92.012320.

[39] G. Agnello, A.N. Cormack, Coordination state and defect evolution in vitreous silica structures formed using Molecular Dynamics under variable cooling conditions, Journal of Non-Crystalline Solids. 451 (2016) 146152. doi:10.1016/j.jnoncrysol.2016.07.003.

[40] X. Li, W. Song, K. Yang, N.M.A. Krishnan, B. Wang, M.M. Smedskjaer, J.C. Mauro, G. Sant, M. Balonis, M. Bauchy, Cooling rate effects in sodium silicate glasses: Bridging the gap between molecular dynamics simulations and experiments, The Journal of Chemical Physics. 147 (2017) 074501. doi:10.1063/1.4998611.

[41] M. Pota, A. Pedone, G. Malavasi, C. Durante, M. Cocchi, M.C. Menziani, Molecular dynamics simulations of sodium silicate glasses: Optimization and limits of the computational procedure, Computational Materials Science. 47 (2010) 739-751. doi:10.1016/j.commatsci.2009.10.017.

[42] N.M.A. Krishnan, B. Wang, Y. Le Pape, G. Sant, M. Bauchy, Irradiation-driven amorphous-to-glassy transition in quartz: The crucial role of the medium-range order in crystallization, Phys. Rev. Materials. 1 (2017) 053405. doi:10.1103/PhysRevMaterials.1.053405.

[43] N.M.A. Krishnan, B. Wang, Y. Yu, Y. Le Pape, G. Sant, M. Bauchy, Enthalpy Landscape Dictates the IrradiationInduced Disordering of Quartz, Physical Review X. 7 (2017) 031019. doi:10.1103/PhysRevX.7.031019.

[44] N.M.A. Krishnan, R. Ravinder, R. Kumar, Y. Le Pape, G. Sant, M. Bauchy, Density-stiffness scaling in minerals upon disordering: Irradiation vs. vitrification, Acta Materialia. 166 (2019) 611-617. doi:10.1016/j.actamat.2019.01.015.

[45] Y. Xiang, J. Du, Effect of Strontium Substitution on the Structure of 45S5 Bioglasses, Chem. Mater. 23 (2011) 2703-2717. doi:10.1021/cm102889q.

[46] M. Wang, B. Wang, N.M.A. Krishnan, Y. Yu, M.M. Smedskjaer, J.C. Mauro, G. Sant, M. Bauchy, lon exchange strengthening and thermal expansion of glasses: Common origin and critical role of network connectivity, Journal of Non-Crystalline Solids. 455 (2017) 70-74. doi:10.1016/j.jnoncrysol.2016.10.027.

[47] Y. Yu, M. Wang, N.M. Anoop Krishnan, M.M. Smedskjaer, K. Deenamma Vargheese, J.C. Mauro, M. Balonis, M. Bauchy, Hardness of silicate glasses: Atomic-scale origin of the mixed modifier effect, Journal of NonCrystalline Solids. 489 (2018) 16-21. doi:10.1016/j.jnoncrysol.2018.03.015. 
[48] S. Plimpton, Fast Parallel Algorithms for Short-Range Molecular Dynamics, Journal of Computational Physics. 117 (1995) 1-19. doi:10.1006/jcph.1995.1039.

[49] N.M.A. Krishnan, Y. Le Pape, G. Sant, M. Bauchy, Effect of irradiation on silicate aggregates' density and stiffness, Journal of Nuclear Materials. 512 (2018) 126-136. doi:10.1016/j.jnucmat.2018.10.009.

[50] J. Du, A.N. Cormack, The medium range structure of sodium silicate glasses: a molecular dynamics simulation, Journal of Non-Crystalline Solids. 349 (2004) 66-79. doi:10.1016/j.jnoncrysol.2004.08.264.

[51] J. Du, Challenges in Molecular Dynamics Simulations of Multicomponent Oxide Glasses, in: Molecular Dynamics Simulations of Disordered Materials, Springer, Cham, 2015: pp. 157-180. doi:10.1007/978-3-31915675-0_7.

[52] W.G. Hoover, Canonical dynamics: equilibrium phase-space distributions, Physical Review A. 31 (1985) 1695.

[53] S. Le Roux, P. Jund, Ring statistics analysis of topological networks: New approach and application to amorphous GeS2 and SiO2 systems, Computational Materials Science. 49 (2010) 70-83. doi:10.1016/j.commatsci.2010.04.023.

[54] D. Massiot, F. Fayon, M. Capron, I. King, S.L. Calvé, B. Alonso, J.-O. Durand, B. Bujoli, Z. Gan, G. Hoatson, Modelling one- and two-dimensional solid-state NMR spectra, Magnetic Resonance in Chemistry. 40 (2002) 70-76. doi:10.1002/mrc.984.

[55] Dependence of the Fictive Temperature of Glass on Cooling Rate - MOYNIHAN - 1976 - Journal of the American Ceramic Society - Wiley Online Library, (n.d.). https://ceramics.onlinelibrary.wiley.com/doi/pdf/10.1111/j.1151-2916.1976.tb09376.x (accessed April 21, 2019).

[56] P.G. Debenedetti, F.H. Stillinger, Supercooled liquids and the glass transition, Nature. 410 (2001) 259-267.

[57] T.F. Middleton, D.J. Wales, Energy landscapes of model glasses. II. Results for constant pressure, The Journal of Chemical Physics. 118 (2003) 4583-4593.

[58] J.C. Mauro, R.J. Loucks, A.K. Varshneya, P.K. Gupta, Enthalpy landscapes and the glass transition, in: S. Yip, T.D. de la Rubia (Eds.), Scientific Modeling and Simulations, Springer Netherlands, 2008: pp. 241-281. http://link.springer.com/chapter/10.1007/978-1-4020-9741-6_15 (accessed August 14, 2016).

[59] H. Liu, S. Dong, L. Tang, N.M. Anoop Krishnan, E. Masoero, G. Sant, M. Bauchy, Long-term creep deformations in colloidal calcium-silicate-hydrate gels by accelerated aging simulations, Journal of Colloid and Interface Science. 542 (2019) 339-346. doi:10.1016/j.jcis.2019.02.022.

[60] Y. Yu, M. Wang, D. Zhang, B. Wang, G. Sant, M. Bauchy, Stretched Exponential Relaxation of Glasses at Low Temperature, Physical Review Letters. 115 (2015) 165901. doi:10.1103/PhysRevLett.115.165901.

[61] J.C. Phillips, Stretched exponential relaxation in molecular and electronic glasses, Rep. Prog. Phys. 59 (1996) 1133-1207. doi:10.1088/0034-4885/59/9/003.

[62] M. Wang, M.M. Smedskjaer, J.C. Mauro, M. Bauchy, Modifier clustering and avoidance principle in borosilicate glasses: A molecular dynamics study, J. Chem. Phys. 150 (2019) 044502. doi:10.1063/1.5051746.

[63] J. Du, L.R. Corrales, First sharp diffraction peak in silicate glasses: Structure and scattering length dependence, Phys. Rev. B. 72 (2005) 092201. doi:10.1103/PhysRevB.72.092201.

[64] S.R. Elliott, Origin of the first sharp diffraction peak in the structure factor of covalent glasses, Physical Review Letters. 67 (1991) 711-714. doi:10.1103/PhysRevLett.67.711.

[65] M. Micoulaut, M. Bauchy, Anomalies of the first sharp diffraction peak in network glasses: Evidence for correlations with dynamic and rigidity properties, Physica Status Solidi (b). 250 (2013) 976-982. doi:10.1002/pssb.201248512.

[66] S.R. Elliott, The origin of the first sharp diffraction peak in the structure factor of covalent glasses and liquids, Journal of Physics: Condensed Matter. 4 (1992) 7661.

[67] P.Y. Huang, S. Kurasch, A. Srivastava, V. Skakalova, J. Kotakoski, A.V. Krasheninnikov, R. Hovden, Q. Mao, J.C. Meyer, J. Smet, D.A. Muller, U. Kaiser, Direct Imaging of a Two-Dimensional Silica Glass on Graphene, Nano Letters. 12 (2012) 1081-1086. doi:10.1021/nl204423x. 
[68] D.S. Brauer, Bioactive glasses-structure and properties, Angewandte Chemie International Edition. 54 (2015) 4160-4181. 